SIAM J. Control and Optimization, vol. 19, No. 4, pp. 445-468, 1981.

Reprints available from hammer@mst.ufl.edu

\title{
CAUSAL FACTORIZATION AND LINEAR FEEDBACK $\dagger$
}

\author{
JACOB HAMMER AND MICHAEL HEYMANN
}

\begin{abstract}
Abstract. An algebraic framework for the investigation of linear dynamic output feedback is introduced. Pivotal in the present theory is the problem of causal factorization, i.e. the problem of factoring two systems over each other through a causal factor. The basic issues are resolved with the aid of the new concept of latency kernels.
\end{abstract}

\section{INTRODUCTION.}

In recent years the system theory literature has seen a rapidly growing interest in questions associated with linear feedback. In the early 1960 's, linear control theory centered chiefly around quadratic (Gaussian) optimal problems and the resulting feedback designs. Later, interest in feedback shifted to a variety of so-called "synthesis" problems. These included the well-known problem of observer design (see Luenberger [1966]), the pole shifting theorem and related issues (Wonham [1967] Simon and Mitter [1968], Brash and Pearson [1970], Heymann [1968]) as well as the decoupling problem (Falb and Wolovich [1967], Gilbert [1969], Wonham and Morse [1970], Morse and Wonham [1970] ). All of these feedback synthesis problems, as well as many others, were formulated and resolved within the framework of state space representations. While most of the work was done with the use of conventional state equations, the work of Wonham and Morse was distinguished by its "coordinate free" setting and initiated what later developed into the celebrated "geometric theory" of linear control (see, e.g., Wonham [1979]).

The current growing interest in linear feedback differs significantly from that of the past both in character and in its source of motivation. While previously the study of feedback was largely oriented at problem solving, the current interest is motivated by a desire of gaining insight into the general nature of linear feedback-chiefly from an algebraic point of view. Much of the motivation for the present trend can be traced back to the work of Rosenbrock [1970] , in which polynomial matrix techniques were used for the study of a variety of (linear) control theoretic questions. Particularly useful turned out to be techniques based on polynomial fraction representations of transfer functions (see, e.g., Heymann [1972], Wolovich [1974], Forney [1975], Fuhrmann [1976]). In this setting of fraction representations, feedback was first studied in Heymann [1972] (see especially Chapter 6 therein), and in a polynomial module framework the study of feedback was initiated by Eckberg [1974]. State feedback also received attention in an algebraic framework by Morse [1975]. A different approach to the study of linear feedback was taken in Hautus and Heymann [1978], where the fundamental underlying object was taken to be the input-output map of the system. There, static linear state feedback was investigated in an algebraic framework consistent with the setting of the (classical) module theory of linear realization as introduced by Kalman (see, e.g., Kalman et al. [1969, Chapter 10] ). More recently, state feedback was also examined in Fuhrmann [1979] using what he termed "polynomial models", and in Münzner and Prätzel-Wolters (1979a], [1979b], [1979c] in a module and category theoretic framework.

While these various approaches to the study of feedback differ from each other substantially both in the underlying concept and in philosophy, they commonly converge on essentially the same (standard) issues that characterize state feedback. It is significant, however, that no success (and, in fact, very little effort, if any) has been reported in respect to output, as opposed to state feedback. When various fundamental questions in regard to output feedback are examined, it becomes immediately clear that difficulties arise that are completely absent in the state-feedback setting. In fact, one discovers immediately that crucial insight is missing. It turns out that the chief reason for this state of affairs is the fact that all of the presently existing algebraic theory of linear systems, and especially that of feedback, rests in one way or another on the theory of modules over the ring $K[z]$ of polynomials and on polynomial matrices. This algebraic machinery is completely satisfactory to develop a fairly comprehensive framework for state feedback. It is not adequate, though, to deal with output-feedback where issues associated with causality become significantly more intricate.

$\dagger$ The latex version of this paper was obtained from the original by using optical character recognition at Mathpix.com. We express our profound thanks to Mathpix. 
The present paper deals in a comprehensive way with the problem of causal output feedback. A related question which receives a great deal of attention in the paper and on which much of the theory hinges is the so-called causal factorization problem. This is the problem of when a given linear input-output map can be factored over another one by a causal linear map. Through the resolution of this issue, questions associated with dynamic causal output feedback are then also resolved. Attention is also given to the static factorization problem as well as the problem of static feedback where special emphasis is placed on the state-feedback case.

A crucial role in the present theory is played by the newly introduced concept of latency. In the discrete time setting, latency expresses "degree of causality" and (intuitively) refers to the intrinsic delay which inputs encounter before output responses are produced. Latency is algebraically expressed by modules over the ring $K\left[\left[z^{-1}\right]\right]$ of power series (in $z^{-1}$ over a field $K$ ). These modules arise in a natural way when the concept of causality is studied algebraically and in fact are readily seen to be the natural algebraic device for the study of feedback.

The paper is organized as follows. In $\S 2$ the basic concepts of $\Lambda K$-linear maps causality, linear i/o maps as well as linear i/s maps, which have been investigated in detail in Hautus and Heymann [1978], are reviewed. The conceptual viewpoint, on which the present investigation of feedback rests, is discussed in $\S 3$. An important technical concept that arises in the algebraic study of linear systems both in connection with the $K[z]$-module theory and the $K\left[\left[z^{-1}\right]\right]$ -module theory is that of "proper bases" and "proper independence". This is the topic of \$4. Section 5 is devoted to the investigation of causal factorization, the main result being Theorem 5.2 and its corollaries. Results are also obtained on static feedback (Theorems 5.10 and 5.14 ). In $\S 6$ the problem of invariants is investigated in detail and explicit characterizations are derived and exhibited. The role of the latency kernels and latency indices is also discussed. The paper is concluded in $\$ 7$ with an investigation of the interesting question of feedback (design) limitations. It is shown that the essential limitation to the possibility of causal feedback implementation of precompensators is the system's latency. In particular, precompensators can be implemented as causal feedback devices modulo a "precompensator remainder" whose dynamic order need not exceed the sum of the system's latency indices.

\section{2. $\Lambda \mathrm{K}$-LINEAR MAPS, CAUSALITY AND INPUT-OUTPUT BEHAVIOR.}

We shall adopt a terminology and setup consistent with that of Hautus and Heymann [1978].

Let $K$ be a field and let $S$ be a $K$-linear space. The class of all truncated $S$-valued Laurent series of the form

$$
\text { (2.1) } s=\sum_{t=t_{0}}^{\infty} s_{t} z^{-t}
$$

is denoted by $S\left(\left(z^{-1}\right)\right)$ or alternatively by $\Lambda S$. The polynomial subset of $S$, i.e., the set of all elements of $\Lambda S$ of the form $\sum_{t \leq 0} s_{t} z^{-t}$, is denoted $\Omega^{+} S$. The power series subset of $\Lambda S$ i.e., the set of all elements of the form $\sum_{t \geq 0} s_{t} z^{-t}$, is denoted $\Omega^{-} S$. The set $\Lambda K=K\left(\left(z^{-1}\right)\right)$ of $K$-valued Laurent series is endowed with a field structure under the operation of convolution as multiplication and coefficientwise addition. In particular, for $\alpha=\sum_{t=t_{0}}^{\infty} \alpha_{t} z^{-t}$ and $\alpha^{\prime}=$ $\sum_{t=t_{i}}^{\infty} \alpha_{t}^{\prime} z^{-t}$ in $\Lambda K$, the product $\alpha \alpha^{\prime}$ is given by

$$
\alpha \alpha^{\prime}=\sum_{t=t_{0}+t_{0}}^{\infty}\left[\sum_{j=t_{0}}^{t-t_{0}^{o}} \alpha_{t} \alpha_{t-j}^{\prime}\right] z^{-t}
$$

and the sum $\alpha+\alpha^{\prime}$ is given by

$$
\alpha+\alpha^{\prime}=\sum_{t=\min ^{\prime}\left(t_{0}, t_{0}^{\prime}\right)}^{\infty}\left(\alpha_{i}+\alpha_{i}^{\prime}\right) z^{-t}
$$

With $\Lambda K$ as the underlying field it then follows that, with convolution as the scalar multiplication and with the usual coefficientwise addition, the set $\Lambda S$ becomes a $\Lambda K$-linear space. When $S$ is a finite dimensional $K$-linear space, say of dimension $n$ then so is $\Lambda S$ as a $\Lambda K$-linear space. It is readily observed that; under the same operations of convolution as multiplication and coefficientwise addition, the field $\Lambda K$ contains (as subobjects) also (i) the ring $K[z]$, or in our notation $\Omega^{+} K$, of polynomials in $z$; (ii) the ring $K\left[\left[z^{-1}\right]\right]$, or in our notation $\Omega^{-} K$, of formal power series in $z^{-1}$; and finally, (iii) the field $K$ itself. It, thus, follows immediately that the set $\Lambda S$ is not only a $\Lambda K$-linear space but is simultaneously also an $\Omega^{+} K$-module, an $\Omega^{-} K$-module and a $K$-linear space As we shall see, these facts turn out to be of central importance in the theory. 
Now, we let $\mathbb{Z}$ denote the integers and for an element $s \in \Lambda S$, given by (2.1), we define the order of $s$ by

$$
\text { (2.2) } \quad \text { ord } s:= \begin{cases}\min \left\{t \in \mathbb{Z} \mid s_{t} \neq 0\right\} & \text { if } s \neq 0 \\ \infty & \text { if } s=0\end{cases}
$$

If $s \neq 0$ and $t_{0}=$ ord $s$, we call the coefficient $s_{t_{0}}$ the leading coefficient of $s$.

Let $U$ and $Y$ be $K$-linear spaces. We shall call $U$ the input value space and $Y$ the output value space of an underlying linear system $\Sigma$. The $\Lambda K$-linear spaces $\Lambda U$ and $\Lambda Y$ are then called the extended input space and extended output space, respectively Elements $u=\Sigma u_{i} z^{-t} \in \Lambda U$ and $y=\Sigma y_{i} z^{-t} \in \Lambda Y$, called, respectively, (extended) inputs and (extended) outputs, are identified with time sequences $\left\{u_{t}\right\}$ and $\left\{y_{t}\right\}$ (with $t$ being identified as time marker).

Let $\bar{f}: \Lambda U \rightarrow \Lambda Y$ be a $K$-linear map. We say that $\bar{f}$ is time invariant if

$$
\bar{f}(z \cdot u)=z \cdot \bar{f}(u)
$$

for all $u \in \Lambda U$, so that $\bar{f}$ is time invariant whenever it is a $\Lambda K$-linear map (Wyman [1972].. Next, for a $\Lambda K$-linear map $\bar{f}: \Lambda U \rightarrow \Lambda Y$ we define the order of $\bar{f}$ by

$$
\text { ord } \bar{f}:=\inf \{\operatorname{ord} \bar{f}(u)-\operatorname{ord} u \mid 0 \neq u \in \Lambda U\}
$$

If the map $\bar{f}$ is the zero map then ord $\bar{f}:=\infty$; otherwise ord $\bar{f}<\infty$. While it is possible that ord $\bar{f}=-\infty$ we shall not concern ourselves here with this case and confine our attention to maps of finite order. This is clearly always the case when $U$ (and hence also $\Lambda U$ ) is finite dimensional.

A $\Lambda K$-linear map $\bar{f}: \Lambda U \rightarrow \Lambda Y$ is called causal if ord $\bar{f} \geqq 0$ and strictly causal if ord $\bar{f}>0$. The map $\bar{f}$ is called order consistent if for each $0 \neq u \in \Lambda U$

$$
\text { ord } \bar{f}(u)-\operatorname{ord} u=\text { ord } \bar{f}
$$

Clearly, an invertible $\Lambda K$-linear map $\bar{l}: \Lambda S \rightarrow \Lambda S$ is order consistent if and only if ord $\bar{l}^{-1}=-$ ord $\bar{l}$. A $\Lambda K$-linear map $\bar{f}$ is said to be order preserving (or instantaneous) if it is order consistent and ord $\bar{f}=0$. An invertible order preserving (and hence causal) $\Lambda K$-linear map $\bar{l}: \Lambda S \rightarrow \Lambda S$ is called $a$ bicausal isomorphism (or simply bicausal) since its inverse is then also causal. Finally, we call $\bar{f}$ nonlatent if it is order consistent and ord $\bar{f}=1$.

We now introduce the following (see also Hautus and Heymann [1978]).

Definition. 2.4. A map $\bar{f}: \Lambda U \rightarrow \Lambda Y$ is called an extended linear input-output map (or extended linear i/o map) if it is strictly causal (i.e., ord $\bar{f}>0$ ) and $\Lambda K$-linear.

Let $L$ denote the $K$-linear space of $K$-linear maps $U \rightarrow Y$ and let $\Lambda L$ denote the $\Lambda K$-linear space of all $L$-Laurent series. We identify this space with the space of $\Lambda K$-linear maps $\Lambda U \rightarrow \Lambda Y$ of finite order as follows. We define the $K$-linear maps

$$
\begin{aligned}
& \bar{\imath}_{u}: U \rightarrow \Lambda U: u \mapsto u \quad \text { (canonical injection) } \\
& \bar{p}_{k}: \Lambda Y \rightarrow Y: \Sigma y_{i} z^{-t} \mapsto y_{k}
\end{aligned}
$$

and with every $\Lambda K$-linear map $\bar{f}: \Lambda U \rightarrow \Lambda Y$ we associate the Laurent series

$$
Z_{\bar{f}}\left(z^{-1}\right):=\Sigma A_{t} z^{-t}
$$

where, for each $k \in \mathbb{Z}$

$$
A_{k}:=A_{k}(\bar{f}):=\bar{p}_{k} \cdot \bar{f} \cdot \bar{\imath}_{u}
$$

The Laurent series (2.6) is called the impulse response or the transfer function of $\bar{f}$. If $u=\Sigma u_{t} z^{-t} \in \Lambda U$ is any element, then the action of $\bar{f}$ on $u$ is given by

$$
\bar{f} \cdot u=\left(\Sigma A_{t}(\bar{f}) z^{-t}\right) \cdot\left(\Sigma u_{t} z^{-t}\right)=\sum_{t} \sum_{k}\left(A_{k}(\bar{f}) u_{t-k}\right) z^{-t}
$$

It is thus immediately seen that

$$
\text { ord } \bar{f}=\min \left\{k \mid A_{k}(\bar{f}) \neq 0\right\},
$$

whence we have the following characterization of causality in terms of the transfer function: The map $\bar{f}$ is causal if and only if $A_{k}(\bar{f})=0$ for $k<0$ and strictly causal if and only if $A_{k}(\bar{f})=0$ for $k \leqq 0$. We also have the following easily verified proposition.

Proposition. 2.10. Let $\bar{f}: \Lambda U \rightarrow \Lambda Y$ be a $\Lambda K$-linear map of order $k_{0}(<\infty)$ and transfer function $Z_{\bar{f}}\left(z^{-1}\right)=$ $\sum_{k=k_{0}}^{\infty} A_{k} z^{-k}$. Then $\bar{f}$ is order consistent if and only if $A_{k_{0}}$ is injective (i.e., $\operatorname{ker} A_{k_{0}}=0$ ).

The following is an immediate corollary to Proposition 2.10. 
Corollary. 2.11. Let $\bar{l}: \Lambda S \rightarrow \Lambda S$ be a causal $\Lambda K$-linear map with transfer function $\sum_{k=0}^{\infty} A_{k}(\bar{l}) z^{-k}$. Then $\bar{l}$ is a bicausal isomorphism if and only if $A_{0}(\bar{l})$ is invertible, in which case $A_{0}\left(\bar{l}^{-1}\right)=\left(A_{0}(\bar{l})\right)^{-1}$.

We associate with an extended linear i/o map $\bar{f}$ a restricted linear i/ o map $\tilde{f}$ which is obtained as follows (see also Hautus and Heymann [1978]). Inputs are restricted to the subset $\Omega^{+} U \subset \Lambda U$, called the restricted input space, and consist of all inputs that terminate at $t=0$, i.e., elements of the form $\Sigma_{t \leq 0} u_{t} z^{-t}$. Outputs are observed only for $t \geqq 1$, that is, in the subset $z^{-1} \Omega^{-} Y$ which is, of course, in bijective correspondence with the $\Omega^{+} K$-quotient module $\Gamma^{+} Y:=\Lambda Y / \Omega^{+} Y$ which we call the restricted output space. The restricted linear i/o map $\tilde{f}: \Omega^{+} U \rightarrow \Gamma^{+} Y$ associated with $\bar{f}$ is then defined by

$$
\tilde{f}=\pi^{+} \cdot \bar{f} \cdot j^{+}
$$

where $j^{+}: \Omega^{+} U \rightarrow \Lambda U$ is the canonical injection and $\pi^{+}: \Lambda Y \rightarrow \Gamma^{+} Y$ is the canonical projection. Clearly, since $\pi^{+}$and $j^{+}$are $\Omega^{+} K$-module homomorphisms, so is also $\tilde{f}$ and we have the following:

Definition. 2.12. A map $\tilde{f}: \Omega^{+} U \rightarrow \Gamma^{+} Y$ is called a restricted linear i/o map if it is an $\Omega^{+} K$-module homomorphism.

Next, we define the linear output response (or output value) map $f: \Omega^{+} U \rightarrow Y$ associated with a given linear i/o map $\bar{f}$ (or $\tilde{f}$ ) as follows:

$$
\text { (2.13) } f: \Omega^{+} U \rightarrow Y: u \mapsto f(u)=\bar{p}_{1} \cdot \bar{f}(u)=p_{1} \cdot \tilde{f}(u)
$$

where (identifying $\Gamma^{+} Y$ with $z^{-1} \Omega^{-} Y$ )

$$
p_{1}: \Gamma^{+} Y \rightarrow Y: \sum_{t=1}^{\infty} y_{t} z^{-t} \mapsto y_{1}
$$

A linear i/o map $\bar{f}$ (or $\tilde{f}$ ) is called reachable if the associated output value map $f$ is surjective.

If $f: \Omega^{+} U \rightarrow Y$ is any $K$-linear map, it can be regarded as an output value map of a linear system. In particular, the restricted and extended linear i/o maps associated with $f$ are then given by

$$
\tilde{f}(u)=\sum_{t \geq 0} f\left(z^{t} u\right) z^{-t-1}, \quad u \in \Omega^{+} U
$$

and

$$
\bar{f}(u)=\sum_{t \in \mathbf{Z}} f\left(\mathcal{S}^{+}\left(z^{t} u\right)\right) z^{-t-1}, \quad u \in \Lambda U
$$

where $\mathcal{S}^{+}: \Lambda U \rightarrow \Omega^{+} U: \Sigma u_{i} z^{-t} \mapsto \sum_{t \leq 0} u_{i} z^{-t}$ is the truncation operator.

The relation between the maps $\bar{f}, \tilde{f}$ and $f$ is summarized by the commutative diagram, Fig. 2.1, in which $i$ denotes the identity map. FIG. 2.1

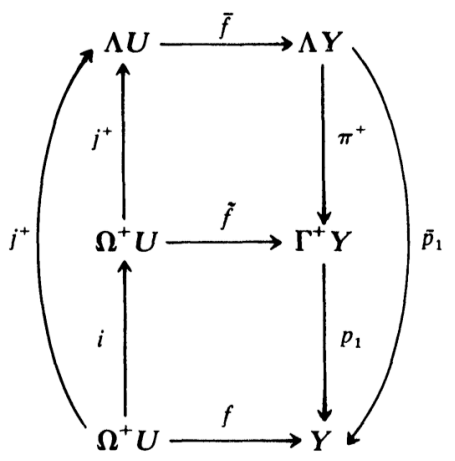

FIGURE 0.1. 2.1

The output value map $f$, which gives for each (restricted) input the value of the output at time $t=1$, is clearly a $K$ -linear map. In some special cases, there exists an $\Omega^{+} K$-module structure on $Y$, compatible with its $K$-vector space structure, such that the output value map $f$ is not just $K$-linear but is also an $\Omega^{+} K$-module homomorphism. When this is the case, then for each $u \in \Omega^{+} U$ and for each positive integer $k, f\left(z^{k} u\right)=z^{k} f(u)$, whence, by (2.15), knowledge 
of the output value at time $t=1$ implies knowledge of the whole ensuing output sequence. This is therefore precisely the case when the system's output "qualifies" as state, a fact which motivates the following definition (for greater detail the reader is referred to Hautus and Heymann [1978]):

Definition. 2.17. An extended linear i/o map $\bar{f}: \Lambda U \rightarrow \Lambda Y$ is called an extended linear input-state (or i/s) map if there exists an $\Omega^{+} K$-module structure on $Y$, compatible with its $K$-linear structure, such that the output value map $f=\bar{p}_{1} \cdot \bar{f} \cdot j^{+}$is an $\Omega^{+} K$ homomorphism. The associated restricted map $\tilde{f}$ is called $a$ restricted linear i/s map.

If $Y$ and $W$ are $K$-linear spaces and $H: Y \rightarrow W$ is a $K$-linear map, then it induces in a natural way a $\Lambda K$-linear map which we call static as follows:

$$
\text { (2.18) } \quad H: \Lambda Y \rightarrow \Lambda W: \Sigma y_{l} z^{-t} \mapsto \Sigma\left(H y_{t}\right) z^{-t}
$$

In a similar way $H$ induces also static $\Omega^{+} K$ and $\Omega^{-} K$-homomorphisms.

We shall need the following characterizations of linear i/s maps, from Hautus and Heymann [1978]

Theorem. 2.19. If $\bar{f}: \Lambda U \rightarrow \Lambda Y$ is an extended linear $i / s$ map then

$$
\text { (2.20) } \operatorname{ker} f=\operatorname{ker} \tilde{f} \text {. }
$$

Theorem. 2.21. Let $\bar{f}: \Lambda U \rightarrow \Lambda Y$ be a reachable extended linear i/o map. Then the following are equivalent:

(i) $\tilde{f}$ is an extended reachable linear i/s map.

(ii) Condition (2.20) holds

(iii) For every extended linear i/o map $\bar{g}: \Lambda U \rightarrow \Lambda W$ satisfying ker $\tilde{f} \subset \operatorname{ker} \tilde{g}$ (where $\tilde{f}$ and $\tilde{g}$ are the corresponding restricted i/o maps and where $W$ is a $K$-linear space there exists a unique static map $H: \Lambda Y \rightarrow \Lambda W$ such that $\bar{g}=H \cdot \bar{f}$.

\section{FEEDBACK AND CAUSAL FACTORIZATION-GENERAL CONSIDERATIONS.}

We shall be concerned with the setup described by the block diagram in Fig. 3.1.

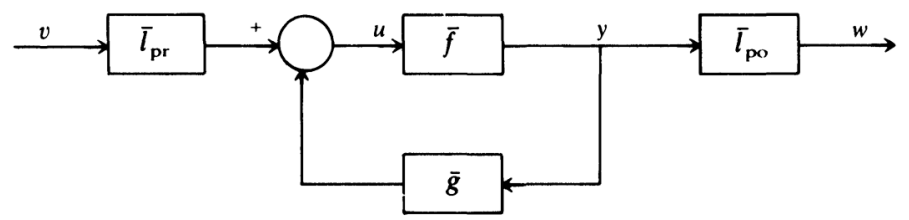

FIGURE 0.2. FIG. 3.1

Here $\bar{f}: \Lambda U \rightarrow \Lambda Y$ is an extended linear i/o map, called the open loop system, $\bar{g}: \Lambda Y \rightarrow \Lambda U$ is a causal $\Lambda K$-linear map called the (output) feedback compensator, $\bar{l}_{\mathrm{pr}}: \underline{\Lambda} U \rightarrow \Lambda U$ is a $\Lambda K$-linear bicausal isomorphism called (bicausal) precompensator and $\bar{l}_{\mathrm{po}}: \Lambda Y \rightarrow \Lambda Y$ is a $\Lambda K$-linear bicausal isomorphism called (bicausal) postcompensator. In case any of the maps $\bar{g}, \bar{l}_{\mathrm{pr}}$ or $\bar{l}_{\mathrm{po}}$ is static we shall call it, respectively a static feedback, pre or post compensator.

Now, since the map $\bar{g}$ is causal and $\bar{f}$ is strictly causal, it readily follows that the composite maps $\bar{f} \cdot \bar{g}: \Lambda Y \rightarrow \Lambda Y$ and $\bar{g} \cdot \bar{f}: \Lambda U \rightarrow \Lambda U$ are both strictly causal. Letting $I$ denote both of the corresponding identity maps, we see that both of the maps $(I+\bar{g} \bar{f}): \Lambda U \rightarrow \Lambda U$ and $(I+\bar{f} \bar{g}): \Lambda Y \rightarrow \Lambda Y$ are bicausal isomorphisms. It follows that the setup of Fig. 3.1 is "well-posed" in the sense that there is a strictly causal $\Lambda K$-linear map $\Lambda U \rightarrow \Lambda Y: v \mapsto w$ given by either of the following composite maps:

$$
\begin{aligned}
& \text { (3.1) } \quad v \mapsto w=\left[\bar{l}_{\mathrm{lo}} \cdot \bar{f} \cdot(I+\bar{g} \bar{f})^{-1} \cdot \bar{l}_{\mathrm{pr}}\right](v) \\
& \text { (3.2) } \quad v \mapsto w=\left[\overline{l_{\mathrm{po}}} \cdot(I+\bar{f} \bar{g})^{-1} \cdot \bar{f} \cdot \bar{l}_{\mathrm{pr}}\right](v)
\end{aligned}
$$

Using again block diagrams, (3.1) and (3.2) can be described, respectively, as in Fig. 3.2a and 3.2b.

In both descriptions, the dashed blocks represent bicausal mappings, so that the compensator configuration of Fig. 3.1 can always be represented equivalently 


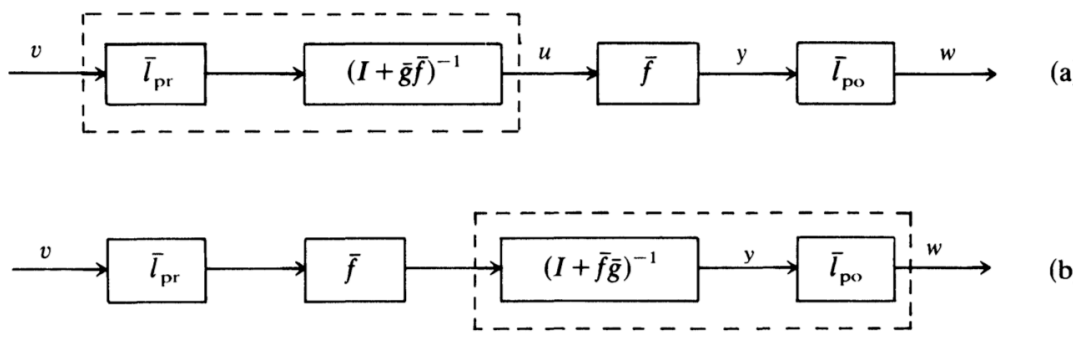

FIGURE 0.3. FIG. 3.2

by the original system preceded and followed by bicausal compensators, with the feedback compensator represented, as one chooses, either as a precompensator or a postcompensator.

Because of the obvious duality between the precompensator situation and the postcompensator situation, there is no need to discuss both of them in detail. since practical interest in postcompensators is at best limited, we shall henceforth confine our attention to precompensation, and discuss postcompensators only in connection with certain mathematical questions.

For various reasons, not to be elaborated on here, feedback compensation is preferred over external compensation whenever possible. Thus, one is interested in the following problem.

Causal feedback problem 3.3. Let $\bar{f}: \Lambda U \rightarrow \Lambda Y$ be an extended linear i/o map.

(a) Under what conditions can a given bicausal $\Lambda K$-linear isomorphism $\bar{l}: \Lambda \dot{U} \rightarrow \Lambda U$ be represented as feedback, i.e. under what conditions do there exist a static map $L: \Lambda U \rightarrow \Lambda U$ and a causal $\Lambda K$-linear map $\bar{g}: \Lambda Y \rightarrow \Lambda Y$, such that $\bar{l}^{-1}=L+\bar{g} \bar{f}$ ?

(b) Under what conditions (on $\bar{f}$ ) can every bicausal $\bar{l}$ be represented as feedback?

Let $\bar{l}: \Lambda U \rightarrow \Lambda U$ be a bicausal $\Lambda K$-linear map, and let

$$
Z_{I^{-1}\left(z^{-1}\right)}=\sum_{t=0}^{\infty} L_{t} z^{-t}
$$

denote the transfer function of $\bar{l}^{-1}$. We can then write

$$
Z_{I^{-1}}\left(z^{-1}\right)=L_{0}+\sum_{t=1}^{\infty} L_{t} z^{-t}=L_{0}+Z_{\bar{h}}\left(z^{-1}\right)
$$

where $L_{0}$ is a static $\Lambda K$-linear map and $Z_{\bar{h}}\left(z^{-1}\right)$ is the transfer function of a strictly causal map $\bar{h}: \Lambda U \rightarrow \Lambda U$ representing the strictly causal part of $\bar{l}^{-1}$. Hence we can always decompose the map $\bar{l}^{-1}$ as

$$
\bar{l}^{-1}=L+\bar{h}
$$

with $L$ static and $\bar{h}$ strictly causal. The causal feedback problem 3.3 is therefore essentially equivalent to the following.

Causal factorization problem 3.4. Let $\bar{f}: \Lambda U \rightarrow \Lambda Y$ be a given strictly causal $\Lambda K$-linear map.

(a) Under what conditions can a strictly causal $\Lambda K$-linear map $\bar{h}: \Lambda U \rightarrow \Lambda U$ be factored causally over $\bar{f}$, i.e., when does there exist a causal map $\bar{g}: \Lambda Y \rightarrow \Lambda U$ such that $\bar{h}=\bar{g} \cdot \bar{f}$ ?

(b) Under what conditions can every strictly causal $\Lambda K$-linear map $\bar{h}: \Lambda U \rightarrow \Lambda U$ be factored causally over $\bar{f}$ ?

It is readily noted that the strict causality of the maps $\bar{f}$ and $\bar{h}$ is inessential to the causal factorization problem, and arises in problem 3.4 only because of the specific requirements of the feedback problem. Indeed, if $\bar{h}$ factors causally over $\bar{f}$, i.e., if there exists a causal $\bar{g}$ such that $\bar{h}=\bar{g} \cdot \bar{f}$, then for each integer $k$ we also have $z^{k} \bar{h}=z^{k} \bar{g} \bar{f}=\bar{g} \cdot\left(z^{k} \bar{f}\right)$ so that $z^{h} \bar{h}$ factors causally over $z^{k} \bar{f}$, and for sufficiently large positive $k$ (unless $\bar{h}$ or $\bar{f}$ are zero) the maps $z^{k} \bar{h}$ and $z^{k} \bar{f}$ are not causal. Thus, the causal factorization problem can be stated in the following less restrictive way:

Given two $\Lambda K$-linear maps $\bar{f}: \Lambda S \rightarrow \Lambda Y$ and $\bar{h}: \Lambda S \rightarrow \Lambda W$ (where $S, Y$ and $W$ are $K$-linear spaces ), when does there exist a causal $\Lambda K$-linear map $\bar{g}: \Lambda Y \rightarrow \Lambda W$ such that the following diagram in Fig. 3.3 commutes

FIG If the causality requirement of $\bar{g}$ is dropped, the factorization problem is standard (see, e.g., Greub [1967]) and $\bar{h}$ factors over $\bar{f}$ if and only if $\operatorname{ker} \bar{f} \subset \operatorname{ker} \bar{h}$. Yet this condition does not say anything about the causality of $\bar{g}$. To deal 


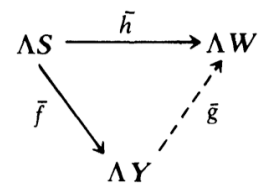

FIGURE 0.4. FIG. 3.3

efficiently with the causality issue, we reintroduce the concept of causality using an approach which is algebraically more tractable.

Let $\bar{f}: \Lambda U \rightarrow \Lambda Y$ be a $\Lambda K$-linear map. We can characterize causality of $\bar{f}$ as follows (compare with our definitions of causality in $\$ 2)$ :

(3.5) The map $\bar{f}$ is causal if and only if $u \in \Omega^{-} U$ implies $\bar{f}(u) \in \Omega^{-} Y$.

Similarly, we have:

(3.6) The map $\bar{f}$ is strictly causal if and only if $u \in z \Omega^{-} U$ implies $\bar{f}(u) \in \Omega^{-} Y$.

Let us denote the $\Omega^{-} K$-quotient module $\Lambda Y / \Omega^{-} Y$ by $\Gamma^{-} Y$, and let $\pi^{-}: \Lambda Y \rightarrow \Gamma^{-} Y$ denote the canonical projection. The following can then be easily verified by the reader.

Proposition. 3.7. Let $\bar{f}: \Lambda U \rightarrow \Lambda Y$ be a $\Lambda K$-linear map.

(a) The map $\bar{f}$ is causal if and only if $\Omega^{-} U \subset \operatorname{ker} \pi^{-} \bar{f}$.

(b) The map $\bar{f}$ is strictly causal if and only if $z \Omega^{-} U \subset \operatorname{ker} \pi^{-} \bar{f}$.

(c) The map $\bar{f}$ is order consistent if and only if, for some integer $k, z^{k} \Omega^{-} U=\operatorname{ker} \pi^{-} \bar{f}$.

(d) The map $\bar{f}$ is instantaneous if and only if $\Omega^{-} U=\operatorname{ker} \pi^{-} \bar{f}$.

(e) The map $\bar{f}$ is nonlatent if and only if $z \Omega^{-1} U=\operatorname{ker} \pi^{-} \bar{f}$.

We shall use the characterizations of the above proposition extensively in the following sections.

\section{PROPER INDEPENDENCE AND PROPER BASES.}

Let $K$ be a field and let $S:=K^{m}$. For an element $0 \neq s \in \Lambda S$, denote by $\hat{s}$ the leading coefficient of $s$. If $s=0$ we shall say that $\hat{s}=0$.

Definition. 4.1. A set of vectors $s_{1}, \cdots, s_{k} \in \Lambda S$ is called properly independent if their leading coefficients $\hat{s}_{1}, \cdots, \hat{s}_{k} \in$ $S$ are $K$-linearly independent.

Below we derive a variety of properties of properly independent sets, of proper bases and of proper direct sum decompositions. Our objective is to develop this theory here only to the extent required in the sequel. Many further results have been omitted, and the reader can, for example, easily verify that the converses of a number of our results are also valid. A more extensive exposition of this and related topics will be published elsewhere

Lemma. 4.2. If $s_{1}, \cdots, s_{k} \in \Lambda S$ is a properly independent set of vectors, then $(i)$ it is $\Lambda K$-linearly independent, and (ii) for every set of scalars $\alpha_{1}, \cdots, \alpha_{k} \in \Lambda K$ the following holds

$$
\text { ord } \sum_{i=1}^{k} \alpha_{i} s_{i}=\min \left\{\operatorname{ord} \alpha_{i} s_{i} \mid i=1, \cdots, k\right\} .
$$

Proof. We shall prove the lemma by showing that if either (i) or (ii) fails to hold then the set $s_{1}, \cdots, s_{k}$ is not properly independent. If $\alpha_{1}, \cdots, \alpha_{k} \in \Lambda K$ is any set of scalars then, by definition, ord $\sum_{i=1}^{k} \alpha_{i} s_{i} \geqq r:=\min \left\{\right.$ ord $\left.\alpha_{i} s_{i} \mid i=1, \cdots, k\right\}$. If either (i) or (ii) fails to hold, there exist $\alpha_{1}, \cdots, \alpha_{k} \in \Lambda K$, not all zero, such that either $\sum_{i=1}^{k} \alpha_{i} s_{i}=0$ or ord $\sum_{i=1}^{k} \alpha_{i} s_{i}>r$. For each $i=1, \cdots, k$ define

$$
\varepsilon_{i}:= \begin{cases}1 & \text { if ord } \alpha_{i} s_{i}=r \\ 0 & \text { if ord } \alpha_{i} s_{i}>r\end{cases}
$$

and consider the terms of order $r$ in $\Sigma \alpha_{i} s_{i}$. This yields $\sum_{i=1}^{k} \varepsilon_{i} \hat{\alpha}_{i} \hat{s}_{i}=0$, implying that $\hat{s}_{1}, \cdots, \hat{s}_{k}$ are $K$-linearly dependent since not all the $\varepsilon_{i} \hat{\alpha}_{i}$ are zero. Hence $s_{1} \cdots, s_{k}$ are not properly independent, completing the proof. 
The condition of Lemma 4.2( ii ) has been called the "predictable degree property in Forney [1975], in the (analogous) setting of "minimal polynomial bases" for rational vector spaces. We shall adopt this terminology and call the property of Lemma 4.2(ii) the predictable order property.

Definition. 4.3. Let $\mathscr{R} \subset \Lambda S$ be a $\Lambda K$-linear subspace. A basis $\left\{s_{1}, \cdots, s_{k}\right\}$ of $\mathscr{R}$ is called proper if the vectors $s_{1}, \cdots, s_{k}$ are properly independent. The basis is called normalized if for each $i=1, \cdots, k$, ord $s_{i}=0$.

To avoid possible confusion in the ensuing discussion where we shall deal with both $K$-linear and $\Lambda K$-linear spaces, we shall use subscripts to emphasize the field. Thus, for example, $\operatorname{span}_{\Lambda K}\left\{s_{1}, \cdots, s_{k}\right\}$ denotes the $\Lambda K$-linear subspace spanned by $s_{1}, \cdots, s_{k} \in \Lambda S$, whereas $\operatorname{span}_{K}\left\{\hat{s}_{1}, \cdots, \hat{s}_{k}\right\}$ denotes the $K$-linear subspace spanned by $\hat{s}_{1}, \cdots, \hat{s}_{k} \in S$. Similarly, $\operatorname{dim}_{\Lambda K} \mathscr{R}$ denotes the dimension of a subspace $\mathscr{R} \subset \Lambda S$ as a $\Lambda \mathrm{K}$-linear space (to distinguish from $K$ -linear). We next have the following theorem.

Theorem. 4.4. Every nonzero $\Lambda K$-linear subspace $\mathscr{R} \subset \Lambda S$ has a proper basis. Moreover, every properly independent subset of $\mathscr{R}$ can be extended to a proper basis.

Proof. Let $0 \neq s_{1} \in \mathscr{R}$ be any vector. Then $s_{1}$ is properly independent. We shall complete the proof by showing that if $s_{1}, \cdots, s_{k} \in \mathscr{R}$ are a properly independent set and if $\mathscr{R}_{k}:=\operatorname{span}_{\Lambda K}\left\{s_{1}, \cdots, s_{k}\right\}$ is a proper subspace of $\mathscr{P}$, we can find a vector $s_{k+1} \in \mathscr{R}$ such that the set $\left\{s_{1}, \cdots, s_{k}, s_{k+1}\right\}$ is also properly independent. The proof is by contradiction. Assume that $\mathscr{R}_{k} \subset \mathscr{R}$ is a proper subspace, let $s_{k+1}^{\circ} \in \mathscr{R}$ be such that the set $\left\{s_{1}, \cdots, s_{k}, s_{k+1}^{\circ}\right\}$ is $\Lambda K$-linearly independent and, without loss of generality, assume that this set is also normalized. Let $\mathscr{P}_{k+1}:=$ $\operatorname{span}_{\Lambda K}\left\{s_{1}, \cdots, s_{k}, s_{k+1}^{\circ}\right\}$ and suppose that there is no vector $s \in \mathscr{R}_{k+1}$ such that the set $\left\{s_{1}, \cdots, s_{k}, s\right\}$ is properly independent. This means that for each $s \in \mathscr{R}_{k+1}, \hat{s} \in \widehat{\mathscr{R}}_{k}:=\operatorname{span}_{K}\left\{\hat{s}_{1}, \cdots, \hat{s}_{k}\right\}$, contradicting, as we shall see, the $\Lambda K$-linear independence of $s_{1}, \cdots, s_{k}, s_{k+1}^{\circ}$. Indeed, we observe that there are scalars $\alpha_{1}^{\circ}, \cdots, \alpha_{k}^{\circ} \in K$ such that $\hat{s}_{k+1}^{\circ}=\sum_{i=1}^{k} \alpha_{i}^{\circ} \hat{s}_{i}$, Let $n_{0}:=0$ and set $s_{k+1}^{1}:=s_{k+1}^{\circ}-\sum_{i=1}^{k} \alpha_{i}^{\circ} z^{-n} s_{i}$, so that ord $s_{k+1}^{1}>$ ord $s_{k+1}^{\circ}$. We now form a sequence of vectors $\left\{s_{k+1}^{t}\right\} t=0,1,2, \cdots$, with $s_{k+1}^{t} \in \mathscr{R}_{k+1}$, such that ord $s_{k+1}^{t+1}>$ ord $s_{k+1}^{t}$ for all $t=0,1,2, \cdots$ as follows: For each $t$, set $n_{t}=$ ord $s_{k+1}^{t}$ and let $s_{k+1}^{r+1}:=s_{k+1}^{t}-\sum_{i=1}^{k} \alpha_{i}^{t} z^{-n} s_{i}$, where the scalars $\alpha_{1}^{t}, \cdots, \alpha_{k}^{t} \in K$ satisfy the condition that $\hat{s}_{k+1}^{t}=\sum_{i=1}^{k} \alpha_{i}^{t} \hat{s}_{i}$. Upon defining $\alpha_{i}:=\sum_{t=0}^{\infty} \alpha_{i}^{t} z^{-n_{i}} \in \Lambda K, \quad i=1, \cdots, k$, it is readily verified that $s_{k+1}^{\circ}-\sum_{i=1}^{k} \alpha_{i} s_{i}=0$ whence $s_{k+1}^{\circ} \in \mathscr{R}_{k}$, a contradiction.

Corollary. 4.5. Let $\mathscr{R} \subset \Lambda S$ be a $\Lambda K$-linear subspace. Then $\operatorname{dim}_{\Lambda K} \mathscr{R}=\operatorname{dim}_{K} \widehat{\mathscr{R}}$ where $\hat{\mathscr{R}}:=\operatorname{span}_{K}\{\hat{s} \mid s \in \mathscr{R}\}$.

Let $\mathscr{R} \subset \Lambda S$ be a $\Lambda K$-linear subspace. If $\mathscr{R}=\mathscr{R}_{1} \oplus \mathscr{R}_{2}$ is a direct sum decomposition of $\mathscr{R}$ into $\Lambda K$-linear subspaces $\mathscr{R}_{1}$ and $\mathscr{R}_{2}$, then, in general, $\hat{\mathscr{R}}_{1} \cap \hat{\mathscr{R}}_{2} \neq 0$ so that $\hat{\mathscr{R}} \neq \hat{\mathscr{R}}_{1}+\hat{\mathscr{R}}_{2}$. This leads us to the following

Definition. 4.6. A direct sum decomposition $\mathscr{R}=\mathscr{R}_{1} \oplus \mathscr{R}_{2}$ of a $\Lambda K$-linear subspace $\mathscr{R} \subset \Lambda S$ into $\Lambda K$-linear subspaces $\mathscr{R}_{1}$ and $\mathscr{R}_{2}$ is called proper if $\hat{\mathscr{R}}_{1} \cap \hat{\mathscr{R}}_{2}=0$ The subspace $\mathscr{P}_{2}$ is then called a proper direct summand of $\mathscr{P}_{1}$,

With the aid of Corollary 4.5 it is readily seen that a direct sum decomposition is proper if and only if $\hat{\mathscr{R}}=\hat{\mathscr{R}}_{1}+\hat{\mathscr{R}}_{2}$. Thus, $\mathscr{R}=\mathscr{R}_{1} \oplus \mathscr{R}_{2}$ is a proper decomposition if and only if there are proper bases $s_{11}, \cdots, s_{1 k_{1}}$ of $\mathscr{R}_{1}$ and $s_{21}, \cdots s_{2 k_{2}}$ of $\mathscr{R}_{2}$ such that the set $s_{11}, \cdots, s_{1 k_{1}}, s_{21}, \cdots, s_{2 k_{2}}$ is a proper basis of $\mathscr{R}$. We then have the following further corollary to Theorem 4.4.

Corollary. 4.7. Let $\mathscr{R} \subset \Lambda S$ be a $\Lambda K$-linear subspace. Then every $\Lambda K$-linear subspace $\mathscr{R}_{1} \subset \mathscr{R}$ has a proper direct summand in $\mathscr{R}$.

Finally, we also have the following variant of the predictable order property.

Corollary. 4.8. Let $\mathscr{R}=\mathscr{R}_{1} \oplus \mathscr{R}_{2}$ be a proper direct sum decomposition of a MK-linear subspace $\mathscr{R} \subset \Lambda S$. Let $s=s_{1}+s_{2}$ be the representation of any vector $s \in \mathscr{R}$, with $s_{i} \in \mathscr{R}_{i}, i=1,2$. Then $\operatorname{ord} s=\min \left\{\operatorname{ord} s_{1}\right.$, ord $\left.s_{2}\right\}$.

Proof. By definition, ord $s \geqq \min \left\{\operatorname{ord} s_{1}\right.$, ord $\left.s_{2}\right\}$. If the above inequality is strict there exist scalars $\alpha_{1}, \alpha_{2} \in K$, not both zero, such that $\alpha_{1} \hat{s}_{1}+\alpha_{2} \hat{s}_{2}=0$ contradicting the fact that $\hat{\mathscr{R}}_{1} \cap \hat{\mathscr{R}}_{2}=0$. 


\section{CAUSAl FACTORIZATION.}

We turn now to the causal factorization problem (3.4). As we mentioned earlier, there is no essential need, in characterizing causal factorizability, to assume strict causality, or even causality, of the maps under consideration. We shall therefore begin with the general case and turn to specific consideration of i/o maps later on. We shall assume that the spaces $U$ and $Y$ are finite dimensional, in particular that $U=K^{m}$ and $Y=K^{p}$. For convenience of notation, we shall temporarily use the notation $\Lambda U$ and $\Lambda Y$ also in connection with $\Lambda K$-linear maps $\bar{f}: \Lambda U \rightarrow \Lambda Y$ that are not necessarily i/o maps (i.e., are not necessarily strictly causal).

Let $\bar{f}: \Lambda U \rightarrow \Lambda Y$ be a $\Lambda K$-linear map and let $\pi^{-}: \Lambda Y \rightarrow \Gamma^{-} Y:=\Lambda Y / \Omega^{-} Y$ be the canonical projection. since $\Omega^{-} Y$ is an $\Omega^{-} K$-module, so is the quotient $\Lambda Y / \Omega^{-} Y$. Thus the map $\pi^{-}$is an $\Omega^{-} K$-homomorphism and so is also the composite $\pi^{-} \bar{f}$. We have

Lemma. 5.1. Let $\bar{f}: \Lambda U \rightarrow \Lambda Y$ be a $\Lambda K$-linear map and let $\pi^{-}: \Lambda Y \rightarrow \Gamma^{-} Y$ be the canonical projection. If $\mathscr{R} \subset$ $\operatorname{ker} \pi^{-} \bar{f}$ is a $\Lambda K$-linear subspace, then $\mathscr{R} \subset \operatorname{ker} \bar{f}$.

Proof. Assume $u \in \mathscr{R} \subset \operatorname{ker} \pi^{-} \bar{f}$, where $\mathscr{R}$ is a $\Lambda K$-linear subspace. Then $\alpha u \in \operatorname{ker} \pi^{-} \bar{f}_{-}$for all $\alpha \in \Lambda K$. Thus $\bar{f}(\alpha u)=\alpha \bar{f}(u) \in \Omega^{-} Y$ for all $\alpha \in \Lambda K$, whence $\bar{f}(u)=0$ and $u \in \operatorname{ker} \bar{f}$ as claimed.

Next we have the following central theorem.

Theorem. 5.2. Let $\bar{f}: \Lambda U \rightarrow \Lambda Y$ and $\bar{h}: \Lambda U \rightarrow \Lambda W$ be $\Lambda K$-linear maps, where $U$ Y and W are finite dimensional $K$-linear spaces. There exists a causal $\Lambda K$-linear map $\bar{g}: \Lambda Y \rightarrow \Lambda W$ such that $\bar{h}=\bar{g} \cdot \bar{f}$ if and only if ker $\pi^{-} \bar{f} \subset \operatorname{ker} \pi^{-} \bar{h}$.

Proof. Suppose $\bar{h}=\bar{g} \cdot \bar{f}$ with $\bar{g}$ causal. Let $u \in \operatorname{ker} \pi^{-} \bar{f}$. Then $\bar{f}(u) \in \Omega^{-} Y$, and by causality of $\bar{g}$ (see Proposition 3.7(a)) $\Omega^{-} Y \subset \operatorname{ker} \pi^{-} \bar{g}$. It follows that $\bar{f}(u) \in \operatorname{ker} \pi^{-} \bar{g}$ whence $u \in \operatorname{ker} \pi^{-} \bar{g} \cdot \bar{f}=\operatorname{ker} \pi^{-} \bar{h}$. Conversely, assume that ker $\pi^{-} \bar{f} \subset \operatorname{ker} \pi^{-} \bar{h}$. By Lemma 5.1 this implies that ker $\bar{f} \subset \operatorname{ker} \bar{h}$ whence by a standard theorem of linear algebra (see, e.g., Greub [1967]) a $\Lambda K$-linear map $\bar{g}: \Lambda Y \rightarrow \Lambda W$ such that $\bar{h}=\bar{g} \cdot \bar{f}$ exists. It remains to be shown that the map $\bar{g}$ can be selected to be causal. To this end write $\Lambda Y=\operatorname{Im} \bar{f} \oplus \mathscr{R}$, where $\operatorname{Im} \bar{f}$ is the image of $\bar{f}$ and $\mathscr{P}$ is any proper direct summand (see Corollary 4.7). Let $\bar{g}_{0}: \Lambda Y \rightarrow \Lambda W$ be any $\Lambda K$-linear map that satisfies the condition that $\bar{h}=\bar{g}_{0} \cdot \bar{f}$ and let $\bar{g}_{1}: \operatorname{Im} \bar{f} \rightarrow \Lambda W$ be the restriction of $\bar{g}_{0}$ to the image of $\bar{f}$. Let $p: \Lambda Y \rightarrow \operatorname{Im} \bar{f}$ denote the projection onto $\operatorname{Im} \bar{f}$ along $\mathscr{P}$; that is, if $y=y_{1}+y_{2} \in \Lambda Y$ is the decomposition of $y$ into its components $y_{1} \in \operatorname{Im} \bar{f}$ and $y_{2} \in \mathscr{R}$, then $p y=y_{1}$. Clearly, $p$ is $\Lambda K$-linear, and we shall see that the map $\bar{g}=\bar{g}_{1} \cdot p$ satisfies the conditions of the theorem. First observe that for $u \in \Lambda U$

$$
\bar{g} \cdot \bar{f}(u)=\bar{g}_{1} \cdot p \bar{f}(u)=\bar{g}_{0} \bar{f}(u)=\bar{h}(u)
$$

so that $\bar{g} \cdot \bar{f}=\bar{h}$. To see that $\bar{g}$ is causal, let $y=y_{1}+y_{2} \in \Omega^{-} Y$, where $y_{1} \in \operatorname{Im} \bar{f}$ and $y_{2} \in \mathscr{R}$. By Proposition 3.7(a), the proof will be complete if we show that $y \in \operatorname{ker} \pi^{-} \bar{g}$ Indeed, Corollary 4.8 implies that both $y_{1}$ and $y_{2}$ are in $\Omega^{-} Y$ so that $\bar{g} \cdot y=\bar{g}_{1} \cdot p y=\bar{g}_{1} \cdot y_{1}=\bar{g}_{0} \cdot \bar{f}(u)$ for some $u \in \operatorname{ker} \pi^{-} \bar{f}$. But by hypothesis ker $\pi^{-} \bar{f} \subset$ ker $\pi^{-} \bar{h}$, whence $\bar{g} \cdot y=\bar{g}_{0} \cdot \bar{f}(u)=\bar{h}(u) \in \Omega^{-} W$ so that $y \in \operatorname{ker} \pi^{-} \bar{g}$ as claimed.

Theorem 5.2 clarifies the significance of the $\Omega^{-} K$-module ker $\pi^{-} \bar{f}$ in connection with the causal factorization problem (and consequently also with feedback). We call this module the latency module or latency kernel of $\bar{f}$.

COROLLARY 5.3. Let $\bar{f}: \Lambda U \rightarrow \Lambda Y$ be a $\Lambda K$-linear map of finite order. Then $\bar{f}$ is order consistent if and only if for every $\Lambda K$-linear map $\bar{h}: \Lambda U \rightarrow \Lambda W$ which satisfies ord $\bar{h} \geqq$ ord $\bar{f}$ there exists a causal $\Lambda K$-linear map $\bar{g}: \Lambda Y \rightarrow \Lambda W$ such that $\bar{h}=\bar{g} \cdot \bar{f}$.

Proof. Recall that a map $\bar{f}$ is order consistent if ord $\bar{f}(u)-$ ord $u=$ ord $\bar{f}$ for each $0 \neq u \in \Lambda U$. Suppose $\bar{f}$ is order consistent and ord $\bar{h} \geqq$ ord $\bar{f}$. Let $0 \neq u \in \operatorname{ker} \pi^{-} \bar{f}$. Then $\bar{f}(u) \in \Omega^{-} Y$ and ord $\bar{f}(u) \geqq 0$. Now ord $\bar{h}(u)-$ ord $u \geqq$ ord $\bar{h} \geqq$ ord $\bar{f}=$ ord $\bar{f}(u)-$ ord $u$ whence ord $\bar{h}(u) \geqq$ ord $\bar{f}(u) \geqq 0$, so that $u \in \operatorname{ker} \pi^{-} \bar{h}$, implying that ker $\pi^{-} \bar{f} \subset \operatorname{ker} \pi^{-} \bar{h}$ By Theorem 5.2 the existence of a causal $\bar{g}$ such that $\bar{h}=\bar{g} \cdot \bar{f}$ is thus assured. Conversely, suppose $\bar{f}$ is not order consistent and that $\bar{h}$ is an order consistent map satisfying ord $\bar{h}=$ ord $\bar{f}$. Then there exists $0 \neq u \in \Lambda U$ such that ord $\bar{f}(u)>$ ord $\bar{f}+$ ord $u=\operatorname{ord} \bar{h}+\operatorname{ord} u=\operatorname{ord} \bar{h}(u)$. If $k:=\operatorname{ord} \bar{f}(u)$, then $0=\operatorname{ord} \bar{f}\left(z^{k} u\right)>\operatorname{ord} \bar{h}\left(z^{k} u\right)$ so that $z^{k} u \in \operatorname{ker} \pi^{-} \bar{f}$ but $z^{k} u \notin \operatorname{ker} \pi^{-} \bar{h}$. Hence ker $\pi^{-} \bar{f} \notin \operatorname{ker} \pi^{-} \bar{h}$ and by Theorem 5.2 there does not exist a causal $\bar{g}$ such that $\bar{h}=\bar{g} \cdot \bar{f}$, completing the proof.

The following corollary which is an immediate consequence of Corollary 5.3 is of central interest in our study of causal factorization since it deals with linear i/o maps and gives us an important characterization of nonlatency.

Corollary. 5.4. Let $\bar{f}: \Lambda U \rightarrow \Lambda Y$ be an extended linear i/o map. Then $\bar{f}$ is nonlatent if and only if for every strictly causal $\Lambda K$-linearmap $\bar{h}: \Lambda U \rightarrow \Lambda W$ there exists a causal $\Lambda K$-linear map $\bar{g}: \Lambda Y \rightarrow \Lambda W$ such that $\bar{h}=\bar{g} \cdot \bar{f}$. 
Let $\bar{f}: \Lambda U \rightarrow \Lambda Y$ be an extended linear i/o map and let $\bar{l}: \Lambda U \rightarrow \Lambda U$ be a bicausal isomorphism, i.e., a bicausal precompensator for $\vec{f}$. Let $\bar{h}$ be the strictly causal part of $\bar{l}^{-1}$, i.e., $\bar{l}^{-1}=L+\bar{h}$ where $L$ is static. As we have seen in $\S 3, \bar{l}$ can be realized as feedback around $\bar{f}$ if $\bar{h}$ factors causally over $\bar{f}$. Theorem 5.2 tells us essentially that the only barrier to realizing a bicausal precompensator as feedback is the relative latency of $\bar{f}$ and $\bar{h}$. Corollary 5.4 characterizes the class of i/o maps over which every bicausal precompensator can be realized as feedback. These i/o maps are, as we have seen, the nonlatent maps (a fact which motivated our choice of terminology). Now, a very special and important class of nonlatent maps is that of injective $\mathrm{i} / \mathrm{s}$ maps. This fact is proved in the following theorem.

Theorem. 5.5. Let $\bar{f}: \Lambda U \rightarrow \Lambda Y$ be an injective linear $i / s$ map. Then $\bar{f}$ is nonlatent.

Proof. By strict causality of $\bar{f}$ we have that $z \Omega^{-} U \subset \operatorname{ker} \pi^{-} \bar{f}$, so that to prove nonlatency we need only to show that ker $\pi^{-1} \bar{f} \subset z \Omega^{-} U$. Let $u \in \operatorname{ker} \pi^{-} \bar{f}$ so that $\bar{f}(u) \in \Omega^{-} Y$. Write $u=u^{+}+u^{-}$, where $u^{+} \in z^{2} \Omega^{+} U$ and $u^{-} \in z \Omega^{-} U$. The proof will be completed by showing that $u^{+}=0$ so that $u \in z \Omega^{-} U$ as claimed. Note that $\bar{f}\left(u^{-}\right) \in \Omega^{-} Y$ by the strict causality of $\bar{f}$ so that, in view of the fact that $\bar{f}(u)=\bar{f}\left(u^{+}\right)+\bar{f}\left(u^{-}\right)$, it follows that $\bar{f}\left(u^{+}\right) \in \Omega^{-} Y$. By (2.16) we have

$$
\bar{f}\left(u^{+}\right)=\sum_{t \in \mathbf{Z}} f\left(\mathcal{S}^{+}\left(z^{i} u^{+}\right)\right) z^{-t-1} \in \Omega^{-} Y,
$$

so that, in particular, $f\left(\mathcal{S}^{+}\left(z^{-2} u^{+}\right)\right)=0$. But $z^{-2} u^{+} \in \Omega^{+} U$, whence $f\left(\mathcal{S}^{+}\left(z^{-2} u^{+}\right)\right)=f\left(z^{-2} u^{+}\right)=0$ implying that $z^{-2} u^{+} \in \operatorname{ker} f=\operatorname{ker} \tilde{f}$ (the equality being a consequence of the i/s property (2.20)). It follows that $\bar{f}\left(z^{-2} u^{+}\right) \in$ $\Omega^{+} Y$, or alternatively, that $\bar{f}\left(u^{+}\right) \in z^{2} \Omega^{+} Y$. since $z^{2} \Omega^{+} Y \cap \Omega^{-} Y=0$, we conclude that $\bar{f}\left(u^{+}\right)=0$ or that $u^{+}=0$ by the injectivity of $\bar{f}$.

While Theorem 5.5 deals only with injective $\mathrm{i} / \mathrm{s}$ maps, it is important to observe that this is not a serious restriction. Indeed, it is shown in Proposition 5.6 below that in the special case of i/s maps (in contrast to i/o maps in general), the kernel is "static"; i.e., if $\bar{f}$ is a noninjective $\mathrm{i} / \mathrm{s}$ map, then $\operatorname{ker} \bar{f}=\Lambda U^{0}$ where $U^{0} \subset U$ is a subspace. This means that the whole degeneracy lies in the input value space $U$ which has been chosen too large, and by restricting the input value space to a proper summand of $U^{0}$ in $U$, the injectivity is restored.

Proposition. 5.6. Let $\bar{f}: \Lambda U \rightarrow \Lambda Y$ be an extended linear $i / s$ map. Then there exists a subspace $U^{0} \subset U$ such that ker $\bar{f}=\Lambda U^{0}$.

Proof. Let $i_{u}: U \rightarrow \Omega^{+} U: u \mapsto u$ be the canonical injection and define the subspace $U^{0} \subset U$ as $U^{0}:=\operatorname{ker} f \cdot i_{u}$, where $f$ is the output value map associated with $\bar{f}$. since $\bar{f}$ is an i/s map we have $\operatorname{ker} f \cdot i_{u}=\operatorname{ker} \tilde{f} \cdot i_{u}=\operatorname{ker} \bar{f} \cdot \bar{i}_{u}$ with the last equality holding by the strict causality of $\bar{f}$. Thus $\bar{i}_{u}\left(U^{0}\right) \subset \operatorname{ker} \bar{f}$, and since $\operatorname{ker} \bar{f}$ is a $\Lambda K$-linear space we conclude that $\Lambda U^{0} \subset \operatorname{ker} \bar{f}$. To prove that ker $\bar{f} \subset \Lambda U^{0}$, it suffices to prove that if $0 \neq u=\sum_{t=t_{0}}^{\infty} u_{t} z^{-t} \in \operatorname{ker} \bar{f}$ then $u_{t_{0}} \in U^{0}$. By recursive application of the same argument this will then imply that $u_{t} \in U^{0}$ for all $t \geqq t_{0}$. Now by formula (2.16) we have $f\left(\mathcal{S}^{+}\left(z^{k} u\right)\right)=0$ for all $k \in \mathbb{Z}$, and since $\mathcal{S}^{+}\left(z^{t_{0}} u\right)=u_{t_{0}}$ the results follow.

The importance of Theorem 5.5 lies in the fact that it tells us that bicausal precompensation is equivalent, in the sense of solvability, to dynamic state feedback Let $\bar{f}: \Lambda U \rightarrow \Lambda Y$ be an extended linear i/o map. We write (see Hautus and Heymann [1978]) $\bar{f}=H \cdot \bar{f}_{s}$, where $H$ is a static output map and $\bar{f}_{s}$ is a reachable i/s map. If $\bar{f}_{s}$ is injective (which is always the case when ker $\bar{f}$ does not contain a subspace of the form $\Lambda S, 0 \neq S \subset U$ ), then every bicausal precompensator can be realized as feedback around $\bar{f}_{s}$. That is, we can write every bicausal $\bar{l}: \Lambda U \rightarrow \Lambda U$ as $\bar{l}^{-1}=L+\bar{g} \bar{f}_{s}$, where $\bar{g}: \Lambda Y \rightarrow \Lambda U$ is a causal $\Lambda K$-linear map and $L$ is static.

Before we proceed with our general investigation, it is worthwhile to record one more consequence of Theorem 5.2.

Corollary. 5.7. Let $\bar{f}_{1}, \bar{f}_{2}: \Lambda U \rightarrow \Lambda Y$ be two extended linear i/o maps with $U$ and $Y$ finite dimensional $K$-linear spaces. There exists a bicausal $\Lambda K$-linearmap $\bar{l}: \Lambda Y \rightarrow \Lambda Y$ such that $\bar{f}_{2}=\bar{l} \cdot \bar{f}_{1}$ if and only if $\operatorname{ker} \pi^{-} \bar{f}_{1}=\operatorname{ker} \pi^{-} \bar{f}_{2}$.

Proof. First, observe that if a bicausal $\bar{l}$ exists then, by Theorem 5.2, it follows immediately that ker $\pi^{-} \bar{f}_{1}=$ ker $\pi^{-} \bar{f}_{2}$. Conversely, assume that $\operatorname{ker} \pi^{-} \bar{f}_{1}=\operatorname{ker} \pi^{-} \bar{f}_{2}$ and write $\Lambda Y=\operatorname{Im} \bar{f}_{1} \oplus \mathscr{R}_{1}=\operatorname{Im} \bar{f}_{2} \oplus \mathscr{R}_{2}$ where $\mathscr{R}_{1}$ and $\mathscr{R}_{2}$ are proper direct summands By Theorem 5,2 there exist causal maps $\bar{l}^{1}, \bar{l}^{2}: \Lambda Y \rightarrow \Lambda Y$ such that $\bar{l}^{1} \bar{f}_{1}=\bar{f}_{2}$ and $\bar{l}^{2} \bar{f}_{2}=\bar{f}_{1}$. Hence $\bar{l}^{2} \cdot \bar{l}^{1} \bar{f}_{1}=\bar{f}_{1}$, and letting $\bar{l}_{1}: \operatorname{Im} \bar{f}_{1} \rightarrow \Lambda Y$ denote the restriction of $\bar{l}^{1}$ to the image of $\bar{f}_{1}$, it is readily verified that $\bar{l}_{1}$ is order preserving. Now, ker $\pi^{-} \bar{f}_{1}=\operatorname{ker} \pi^{-} \bar{f}_{2}$ implies that $\operatorname{ker} \bar{f}_{1}=\operatorname{ker} \bar{f}_{2}$, whence $\operatorname{dim} \operatorname{Im} \bar{f}_{1}=\operatorname{dim} \operatorname{Im} \bar{f}_{2}$ and $\operatorname{dim} \mathscr{R}_{1}=\operatorname{dim} \mathscr{R}_{2}$. Let $\bar{l}_{2}: \mathscr{R}_{1} \rightarrow \Lambda Y$ be an order preserving map satisfying $\operatorname{Im} \bar{l}_{2}=\mathscr{R}_{2}$ and let $p: \Lambda Y \rightarrow \operatorname{Im} \bar{f}_{1}$ denote the projection along $\mathscr{R}_{1}$. We claim that the map $\bar{l}: \Lambda Y \rightarrow \Lambda Y: y \mapsto \bar{l}_{1} p y+\bar{l}_{2}(I-p) y$ is a bicausal isomorphism and that $\bar{l} \cdot \bar{f}_{1}=\bar{f}_{2}$. Indeed, to see the latter property, note that for any $u \in \Lambda U$ we have

$$
\bar{l} \bar{f}_{1}(u)=\bar{l}_{1} p \bar{f}_{1}(u)+\bar{l}_{2}(I-p) \bar{f}_{1}(u)=\bar{l}_{1} \cdot \bar{f}_{1}(u)=\bar{l}^{1} \bar{f}_{1}(u)=\bar{f}_{2}(u) .
$$


To see the bicausality of $\bar{l}$ it suffices to show that it is order preserving. Indeed, let $y=y_{1}+y_{2} \in \Lambda Y$ be any element with $y_{1} \in \operatorname{Im} \bar{f}_{1}$ and $y_{2} \in \mathscr{R}_{1}$. Then $\bar{l} y=\bar{l}_{1} y_{1}+\bar{l}_{2} y_{2}$ and using Corollary 4.8 together with the fact that $\operatorname{Im} \bar{l}_{1}$ and $\operatorname{Im} \bar{l}_{2}$ form a proper direct sum, we have that ord $\bar{l} y=\min \left\{\operatorname{ord} \bar{l}_{1} y_{1}\right.$, ord $\left.\bar{l}_{2} y_{2}\right\}=\min \left\{\operatorname{ord} y_{1}\right.$, ord $\left.y_{2}\right\}$, where the last equality follows from the order preserving property of $\bar{l}_{1}$ and $\bar{l}_{2}$. Using Corollary 4.8 again, together with the fact that $\operatorname{Im} \bar{f}_{1}$ and $\mathscr{R}_{1}$ form a proper direct sum, gives that $\min \left\{\right.$ ord $y_{1}$, ord $\left.y_{2}\right\}=$ ord $y$ whence ord $\bar{l} y=$ ord $y$ as claimed and the proof is complete.

Clearly, the bicausal $\Lambda K$-linear map $\bar{l}$ of Corollary 5.7 can be regarded as a bicausal postcompensator for $\bar{f}_{1}$, and there is a kind of duality between feedback and compensation which deserves some further comments.

Let $\bar{f}: \Lambda U \rightarrow \Lambda Y$ be an extended linear i/o map and let $\bar{l}_{\mathrm{pr}}: \Lambda U \rightarrow \Lambda U$ be a bicausal precompensator for $\bar{f}$. If $\bar{w}: \Lambda U \rightarrow \Lambda U$ is the strictly causal part of $\bar{l}_{\mathrm{pr}}$, then the causal feedback problem is that of existence of a causal $\Lambda K$ -linear map $\bar{g}: \Lambda Y \rightarrow \Lambda U$ such that $\bar{w}=\bar{g} \cdot \bar{f}$. The map $\bar{g}$ can be regarded essentially as a causal (but not necessarily bicausal) postcompensator for $\bar{f}$. Conversely, if $\bar{l}_{\mathrm{po}}: \Lambda Y \rightarrow \Lambda Y$ is a bicausal postcompensator and if $\bar{w}: \Lambda Y \rightarrow \Lambda Y$ the strictly causal part of $\bar{l}_{\text {po }}$, the dual of the above causal factorization problem is that of the existence of a causal $\Lambda K$ -linear map $\bar{g}: \Lambda Y \rightarrow \Lambda U$ such that $\bar{w}=\bar{f} \cdot \bar{g}$. Here $\bar{g}_{-}$can be viewed as a causal, but again not necessarily bicausal, precompensator for $\bar{f}$. Thus the pre- and postcompensator problems become interrelated through feedback. We can also write down the dual of Corollary 5.7 regarding the problem of bicausal precompensation.

Corollary. COROLLARY 5.8. Let $\bar{f}_{1}, \bar{f}_{2}: \Lambda U \rightarrow \Lambda Y$ be two extended linear i/o maps with $U$ and $Y$ finite dimensional $K$-linear spaces. There exists a bicausal $\Lambda K$-linearmap $\bar{l}: \Lambda U \rightarrow \Lambda U$ such that $\bar{f}_{2}=\bar{f}_{1} \cdot \bar{l}$ if and only if ker $\pi^{-} \bar{f}_{1}^{*}=\operatorname{ker} \pi^{-} \bar{f}_{2}^{*}$, where $\bar{f}_{1}^{*}$ and $\bar{f}_{2}^{*}$ denote the dual maps of $\bar{f}_{1}$ and $\bar{f}_{2}$ respectively.

In Corollary 5.8 the dual maps $\bar{f}_{1}^{*}$ and $\bar{f}_{2}^{*}$ can of course be identified with the transposes of the corresponding maps (or transfer functions) in view of the finite dimensionality of the underlying spaces.

In Hautus and Heymann [1978], the static state feedback problem was investigated. This is the following problem: Given an extended linear i/s map $\bar{f}: \Lambda U \rightarrow \Lambda Y$ under what conditions can a bicausal precompensator $\bar{l}: \Lambda U \rightarrow \Lambda U$ be written as $\bar{l}^{-1}=L+G \bar{f}$, where $L$ and $G$ are static maps. It was shown there that a necessary and sufficient condition for the static state feedback problem to have a solution is that

$$
(5.9) \bar{l}^{-1}(\operatorname{ker} \tilde{f}) \subset \Omega^{+} U
$$

where $\tilde{f}: \Omega^{+} U \rightarrow \Gamma^{+} Y$ is the restricted i/s map associated with $\bar{f}$. We now turn to the more general question of static output (rather than state) feedback. As we have been doing throughout this paper, we focus our attention on the static factorization problem which is characterized in the following

Theorem. 5.10. Let $\bar{f}: \Lambda U \rightarrow \Lambda Y$ and $\bar{h}: \Lambda U \rightarrow \Lambda W$ be $\Lambda K$-linear maps. There exists a static $\Lambda K$-linear map $G: \Lambda Y \rightarrow \Lambda W$ such that $\bar{h}=G \cdot \bar{f}$ if and only if $\operatorname{ker} \bar{p}_{1} \cdot \bar{f} \subset \operatorname{ker} \bar{p}_{1} \cdot \bar{h}$.

Proof. Assume first that $G$ exists so that $\bar{h}=G \cdot \bar{f}$. Then $u \in \operatorname{ker} \bar{p}_{1} \cdot \bar{f}$ implies that $\bar{p}_{1} \cdot \bar{f}(u)=0$, whence $\bar{p}_{1} \cdot \bar{h}(u)=\bar{p}_{1} \cdot G \cdot \bar{f}(u)=G \cdot \bar{p}_{1} \cdot \bar{f}(u)=0$, so that $u \in \operatorname{ker} \bar{p}_{1} \cdot \bar{h}$ Conversely, assume that $\operatorname{ker}_{1} \cdot \bar{f} \subset \operatorname{ker} \bar{p}_{1} \cdot \bar{h}$. This implies the existence of a $K$-linear map $G: Y \rightarrow W$ such that $\bar{p}_{1} \cdot \hbar=G_{-} \cdot \bar{p}_{1} \cdot \bar{f}$. By definition of static maps (see (2.18)), we have that $G \cdot \bar{p}_{1}=\bar{p}_{1} \cdot G$ so that $\bar{p}_{1}(\bar{h}-G \cdot \bar{f})=0$. That this implies $\bar{h}=G \bar{f}=0$ is seen as follows. Suppose to the contrary that $(\bar{h}-G \cdot \bar{f})(u)=\sum_{t \in Z} y_{l} z^{-t} \neq 0$ for some $u \in \Lambda U$ Then there exists $k \in \mathbb{Z}$ such that $y_{k} \neq 0$. Let $\hat{u}=z^{k-1} u$ and note that $p_{1}(\bar{h}-G \bar{f})(\hat{u})=p_{1} \sum_{\epsilon \in \mathbf{Z}} y_{i} z^{-i+k-1}=y_{k} \neq 0$, a contradiction.

We shall conclude the present discussion by specializing our static factorization results to the case of linear i/s maps. We need the following lemma.

Lemma. 5.11. Let $\bar{f}: \Lambda U \rightarrow \Lambda Y$ be an injective extended linear $i / s$ map. Then $\operatorname{ker} \pi^{+} \bar{f} \subset \Omega^{+} U$.

Proof. Let $u \in \operatorname{ker} \pi^{+} \bar{f}$ be any element. Then $\bar{f}(u) \in \Omega^{+} Y$ so that $\bar{p}_{1} \cdot \bar{f}(u)=0$ Write $u=u^{+}+u^{-}$, where $u^{+} \in \Omega^{+} U$ and $u^{-} \in z^{-1} \Omega^{-} U$. Then by the strict causality of it follows that $\bar{f}\left(u^{-}\right) \in z^{-2} \Omega^{-} Y$ and $\bar{p}_{1} \cdot \bar{f}\left(u^{-}\right)=0$. Hence $\bar{p}_{1} \cdot \bar{f}\left(u^{+}\right)=\bar{p}_{1} \cdot \bar{f}(u)-\bar{p}_{1} \cdot \bar{f}\left(u^{-}\right)=0$ and $u_{-}^{+} \in \operatorname{ker} \bar{p}_{1} \cdot \bar{f} \cdot j^{+}=\operatorname{ker} f=\operatorname{ker} \tilde{f}$, the last equality follow ing from the i/s property of $\bar{f}$. We conclude that $\bar{f}\left(u^{+}\right) \in \Omega^{+} Y$ so that also $\bar{f}\left(u^{-}\right)=\bar{f}(u)-\bar{f}\left(u^{+}\right) \in \Omega^{+} Y$. Hence $\bar{f}\left(u^{-}\right) \in \Omega^{+} Y \cap z^{-2} \Omega^{-} Y=0$ and, by the injectivity of $\bar{f}, u^{-}=0$ concluding the proof.

Corollary. 5.12. Let $\bar{f}: \Lambda U \rightarrow \Lambda Y$ be an injective extended linear $i / s$ map and let $\bar{h}: \Lambda U \rightarrow \Lambda W$ be a strictly causal $\Lambda K$-linear map. Then there exists a static map $G: \Lambda Y \rightarrow \Lambda W$ such that $\bar{h}=G \cdot \bar{f}$ if and only if ker $\pi^{+} \bar{f} \subset \operatorname{ker} \pi^{+} \bar{h}$. 
Proof. If $G$ exists such that $\bar{h}=G \cdot \bar{f}$, then $u \in \operatorname{ker} \pi^{+} \bar{f}$ implies that $\bar{f}(u) \in \Omega^{+} Y$, so that $\bar{h}(u)=G \cdot \bar{f}(u) \in \Omega^{+} W$ and $u \in \operatorname{ker} \pi^{+} \hbar$. Conversely, suppose $\operatorname{ker} \pi^{+} \bar{f} \subset \operatorname{ker} \pi^{+} \bar{h}$ We will show that this implies that $\operatorname{ker} \bar{p}_{1} \cdot \bar{f} \subset \operatorname{ker} \bar{p}_{1} \cdot \bar{h}$, from which the existence of $G$ is insured by Theorem 5.10. Let $u \in \operatorname{ker} \bar{p}_{1} \cdot \bar{f}$ be any element and write $u=u^{+}+u^{-}$ where $u^{+} \in \Omega^{+} U$ and $u^{-} \in z^{-1} \Omega^{-} U$. Then, by strict causality of both $\bar{f}$ and $\bar{h}$ it follows that $\bar{f}\left(u^{-}\right) \in z^{-2} \Omega^{-} Y$ and $\bar{h}\left(u^{-}\right) \in z^{-2} \Omega^{-} W$ yielding $\bar{p}_{1} \bar{f}\left(u^{-}\right)=0$ and $\bar{p}_{1} \bar{h}\left(u^{-}\right)=0$ Hence, $u^{+}=u-u^{-} \in \operatorname{ker} \bar{p}_{1} \bar{f}$ so that $u^{+} \in \operatorname{ker} f=\operatorname{ker} \hat{f}$, the last equality following from the i/s property of $\bar{f}$. Consequently $u^{+} \in \operatorname{ker} \tilde{f} \subset \operatorname{ker} \pi^{+} \bar{f} \subset \operatorname{ker} \pi^{+} \bar{h} \subset \operatorname{ker} \bar{p}_{1} \bar{h}$, the last inclusion holding by definition. Thus $u=u^{+}+u^{-} \in \operatorname{ker} \bar{p}_{1} \bar{h}$, and the proof is complete.

Let $\bar{f}: \Lambda U \rightarrow \Lambda Y$ be a reachable linear i/s map. Let $\bar{l}: \Lambda U \rightarrow \Lambda U$ be a bicausal isomorphism and write $\bar{l}^{-1}=L+h$, where $L$ is static and $\bar{h}$ is strictly causal. Corollary 5.12 can then be interpreted as a solvability condition of the static state feedback problem. Clearly, the condition of the corollary must be equivalent with condition (5.9) which was obtained in Hautus and Heymann [1978]. We shall see next (Theorem 5.14 below) that this is indeed the case. We require the following lemma.

Lemma. 5.13. Let $\bar{f}: \Lambda U \rightarrow \Lambda Y$ be an extended linear $i / s$ map and let $\bar{h}: \Lambda U \rightarrow \Lambda W$ be a strictly causal $\Lambda K$ -linear map. Then ker $\tilde{f} \subset \operatorname{ker} \tilde{h}$ only if $\operatorname{ker} \bar{f} \subset \operatorname{ker} \bar{h}$

Proof. Assume that ker $\bar{f} \not \subset \operatorname{ker} \bar{h}$ and let $u \in \operatorname{ker} \bar{f}$ satisfy $\bar{h}(u) \neq 0$. Then there exists $k \in \mathbb{Z}$ such that $\pi^{+} \bar{h}\left(z^{k} u\right) \neq$ 0 so that by the strict causality of $\bar{h}$ we have that $0 \neq \mathcal{S}^{+}\left(z^{k} u\right) \in \Omega^{+} U$ and $\pi^{+} \bar{h}\left(\mathcal{S}^{+}\left(z^{k} u\right)\right)=\tilde{h}\left(\mathcal{S}^{+}\left(z^{k} u\right)\right) \neq$ 0. However, $\bar{f}\left(z^{k} u\right)=0$ and upon application of Proposition 5.6 we also have that $\bar{f}\left(\mathcal{S}^{+}\left(z^{k} u\right)\right)=0$, whence $\mathcal{S}^{+}\left(z^{k} u\right) \in \operatorname{ker} \tilde{f}$. Thus $\operatorname{ker} \hat{f} \notin \operatorname{ker} \tilde{h}$ and the proof is complete.

Theorem. 5.14. Let $\bar{f}: \Lambda U \rightarrow \Lambda Y$ be a reachable extended linear i/s map. Let $\bar{l}: \Lambda U \rightarrow \Lambda U$ be a bicausal $\Lambda K$ -linear map and write $\bar{l}^{-1}=L+\bar{h}$ where $L$ is static and $\bar{h}$ is strictly causal. Then $\operatorname{ker} \pi^{+} \bar{f} \subset \operatorname{ker} \pi^{+} \bar{h}$ if and only if $\bar{l}^{-1}(\operatorname{ker} \tilde{f}) \subset \Omega^{+} U$.

Proof. Suppose ker $\pi^{+} \bar{f} \subset \operatorname{ker} \pi^{+} \bar{h}$. Let $u \in \operatorname{ker} \tilde{f}$ be any element. Then $u \in \operatorname{ker} \pi^{+} \bar{h}$, and since $u \in \Omega^{+} U$ we also have that $u \in \operatorname{ker} \pi^{+} L$. Hence $u \in\left(\operatorname{ker} \pi^{+} \bar{h}\right) \cap\left(\operatorname{ker} \pi^{+} L\right) \subset \pi^{+}(\bar{h}+L)=\operatorname{ker} \pi^{+} \bar{l}^{-1}$ so that $\bar{l}^{-1}(u) \in \Omega^{+} U$. Conversely assume that $\bar{l}^{-1}(\operatorname{ker} \tilde{f}) \subset \Omega^{+} U$. This immediately implies that ker $\tilde{f} \subset$ ker $\tilde{h}$ whence, by Lemma 5.13, ker $\bar{f} \subset \operatorname{ker} \bar{h}$. Now let $u \in \operatorname{ker} \pi^{+} \bar{f}$ and write $u=u^{+}+u^{-}$with $u^{+} \in \Omega^{+} U$ and $u^{-} \in z^{-1} \Omega^{-} U$. Then $\bar{f}\left(u^{-}\right) \in z^{-2} \Omega^{-} Y$, and since $\bar{f}(u) \in \Omega^{+} Y$ we conclude that $\bar{p}_{1} \cdot \bar{f}\left(u^{+}\right)=0$. This implies that $u^{+} \in \operatorname{ker} f=\operatorname{ker} \tilde{f}$ (with the equality holding since $\bar{f}$ is an i/s map) so that $u^{+} \in \operatorname{ker} \tilde{h} \subset \operatorname{ker} \pi^{+} \bar{h}$. Finally, $u^{+} \in \operatorname{ker} \tilde{f}$ implies that $\bar{f}\left(u^{+}\right) \in \Omega^{+} U$ whence $\bar{f}\left(u^{-}\right)=\bar{f}(u)-\bar{f}\left(u^{+}\right) \in \Omega^{+} Y$. But then $\bar{f}\left(u^{-}\right) \in \Omega^{+} Y \cap z^{-2} \Omega^{-} Y=0$, so that $u^{-} \in \operatorname{ker} \bar{f} \subset \operatorname{ker} \bar{h}$, and hence $u^{-} \in \operatorname{ker} \pi^{+} \bar{h}$. This implies that $u=u^{+}+u^{-} \in \operatorname{ker} \pi^{+} \bar{h}$ concluding the proof.

\section{FACTORIZATION INVARIANTS- EXPLICIT CALCULATION.}

Throughout this section we shall assume that $U=K^{m}$ and $Y=K^{p}$, and we shall study properties of $\Lambda U$ as an $\Omega^{-} K$-module as well as properties of submodules thereof

The ring $\Omega^{-} K$ is of course a principal ideal domain, and clearly also a Euclidean domain. The units of $\Omega^{-} K$ are precisely those elements whose order is zero and each element $0 \neq \alpha \in \Omega^{-} K$ can be expressed as

$$
\alpha=z^{-\operatorname{ord} \alpha} \alpha_{0}
$$

where $\alpha_{0} \in \Omega^{-} K$ is a unit. It is clear, therefore, that all the ideals of $\Omega^{-} K$ are of the form $\left(z^{-k}\right)$, forming a chain with $\left(z^{-1}\right)$ being the unique maximal ideal and the only prime. Thus, the ring $\Omega^{-} K$ is also a local ring and $\Omega^{-} K /\left(z^{-1}\right)$ is a field, isomorphic to the field $\mathscr{K}_{0}$ which consists of the units of $\Omega^{-} K$ augmented by zero. We shall make use of the special properties of the ring $\Omega^{-} K$ in the ensuing discussion.

For a fixed integer $k$, consider the subset $z^{-k} \Omega^{-} U \subset \Lambda U$. Clearly, this subset is an $\Omega^{-} K$ submodule of $\Lambda U$. Moreover, while $\Lambda U$ itself is not a finitely generated $\Omega^{-} K$ module, the submodule $z^{-k} \Omega^{-} U$ is (and hence is a free module). In fact, it is readily noted that $\operatorname{rank}_{\Omega^{-} K} z^{-k} \Omega^{-} U=\operatorname{dim}_{\Lambda K} \Lambda U=\operatorname{dim}_{K} U$. Indeed, if $\left\{e_{1}, \cdots, e_{m}\right\}$ is a basis for $U$ (as well as for $\Lambda U$ ), then $\left\{z^{-k} e_{1}, \cdots, z^{-k} e_{m}\right\}$ is a basis (i.e., a free generator) for $z^{-k} \Omega^{-} U$.

Let $0 \neq \Delta \subset \Lambda U$ be an $\Omega^{-} K$-submodule. We say that $\Delta$ is of finite order if there exists a finite integer $k$ such that $\Delta \subset z^{-k} \Omega^{-} U$. The maximal integer $k$ for which the above holds, and which is the least order of elements in $\Delta$, is denoted $k_{\Delta}$ and is called the order of $\Delta$. We define the order of the zero module as infinity. We have the following:

Proposition. 6.1. Let $0 \neq \Delta \subset \Lambda U$ be an $\Omega^{-} K$-submodule. Then $\Delta$ is finitely generated if and only if it has finite order. 
Proof. If $\Delta$ has finite order there exists a finite integer $k$ such that $\Delta$ is a submodule of $z^{-k} \Omega^{-} U$ which is, of course, finitely generated. since $\Omega^{-} K$ is a principal ideal domain, $\Delta$ is then also finitely generated. Conversely, if $\Delta$ is finitely generated, say by elements $d_{1}, \cdots, d_{m} \in \Delta$, then clearly $\Delta \subset z^{-k_{2}} \Omega^{-} U$, where $k_{\Delta}:=\min \left\{\operatorname{ord} d_{i}, i=1, \cdots, m\right\}$.

Let $\Delta \subset \Lambda U$ be a finitely generated $\Omega^{-} K$-submodule. Then, by Proposition 6.1 , it is of finite order and hence rank $\Delta \leqq \operatorname{dim} U(=m)$. Let $\Delta$ be of rank $n$ and let $d_{1}, \cdots, d_{n}$ be a basis for $\Delta$. Define the $\Omega^{-} K$-homomorphism $D$ : $\Omega^{-} K^{n} \rightarrow \Delta$ by $D e_{i}=d_{i}, i=1, \cdots, n$, where $e_{1}, \cdots, e_{n}$ denotes the natural basis for $K^{n}$ (as well as for $\Omega^{-} K^{n}$ ). We can view $D$ also as a matrix with entries in $\Lambda K$ by regarding $d_{i} \in \Lambda K^{m}(=\Lambda U)$ as the $i$ th column of $D$. Conversely, if $D$ is an $m \times n$ matrix with entries in $\Lambda K$, we can regard $D$ as an $\Omega^{-} K$-homomorphism $\Omega^{-} K^{n} \rightarrow \Lambda U: e_{i} \mapsto d_{i}, i=1, \cdots, n$, where $d_{i} \in \Lambda U$ is the $i$ th column of $D$. The image $\Delta=D \Omega^{-} K^{n}:=\left\{D w \mid w \in \Omega^{-} K^{n}\right\}$ is an $\Omega^{-} K$-submodule of $\Lambda U$ Clearly, rank $\Delta=\operatorname{rank} D$, where rank $D$ is the matrix rank of $D$ over the ring $\Omega^{-} K$ (or over $\Lambda K$ ).

Consider now the special case when $n=m$ (that is, $K^{m}=U$ ) and let $D$ be a nonsingular $m \times m$ matrix with entries in $\Lambda K$. Then $D$ defines, as above, an $\Omega^{-} K$ homomorphism $\Omega^{-} U \rightarrow \Lambda U$ and also (when simply regarded as a transfer function) a $\Delta K$-linear map $\Lambda U \rightarrow \Lambda U$. Denoting both maps by the same symbol $D$, it is readily verified that the diagram in Fig. 6.1 is commutative,

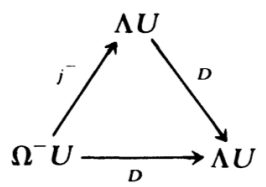

FIGURE 0.5. FIG. 6.1

where $j^{-}$denotes the canonical injection. since the matrix $D$ is nonsingular, the $\Lambda K$-linear map $D$ is invertible. We shall say that the matrix $D$ is bicausal if the associated $\Lambda K$-linear map is bicausal, i.e., if the entries of $D$ are in $\Omega^{-} K$ and its determinant is a unit in this ring (that is, has order zero). In analogy we shall say that a matrix $D$ is strictly causal or causal if so is the associated $\Lambda K$-linear map. Finally, an $\Omega^{-} K$-submodule $\Delta=D \Omega^{-} U \subset \Lambda U$ is called a full submodule if rank $\Delta=m$, i.e., if the matrix $D$ is nonsingular.

Theorem. 6.2. Let $\Delta_{1}, \Delta_{2} \subset \Lambda U$ be finitely generated $\Omega^{-} K$-submodules given by $\Delta_{1}=D_{1} \Omega^{-} U$ and $\Delta_{2}=D_{2} \Omega^{-} U$. Then $\Delta_{2} \subset \Delta_{1}$ if and only if there exists a causal matrix $R$ (i.e., with entries in $\Omega^{-} K$ ) such that $D_{2}=D_{1} R$.

The proof of Theorem 6.2 is elementary and will be omitted. The following corollary will be useful in the sequel.

Corollary. 6.3. Let $\Delta_{1}, \Delta_{2} \subset \Lambda U$ be finitely generated $\Omega^{-} K$-submodules given by $\Delta_{1}=D_{1} \Omega^{-} U$ and $\Delta_{2}=D_{2} \Omega^{-} U$. Assume that $\Delta_{1}$ is full and define $R:=D_{1}^{-1} D_{2}$. Then $\Delta_{2} \subseteq \Delta_{1}$ if and only if $R$ is causal with equality if and only if $R$ is bicausal.

Let $\Delta \subset \Lambda U$ be a finitely generated $\Omega^{-} K$-submodule of rank $n$ and order $k_{\Delta}$. Then for all integers $j \leqq k_{\Delta}, \Delta \subset$ $z^{-j} \Omega^{-} U$ and for each integer $j \geqq k_{\Delta}$ we define the submodule $\Delta_{j} \subset \Delta$ by

$$
\Delta_{i}:=\Delta \cap z^{-i} \Omega^{-} U .
$$

Clearly $z^{-i} \Omega^{-} U \subset z^{-k} \Omega^{-} U$ for all $j \geqq k$, and it follows that

$$
\text { (6.5) } \Delta=\Delta_{k_{\Delta}} \supset \Delta_{k_{2}+1} \supset \cdots>\Delta_{i} \supset \Delta_{j+1} \cdots
$$

As an immediate consequence of the fact that if $u \in \Delta_{i}$ then $z^{-1} u \in \Delta_{j+1}$, it is clear that rank $\Delta=\operatorname{rank} \Delta_{j}$ for all $j$ and the quotient modules

$$
\mathscr{D}_{i}:=\Delta_{i} / \Delta_{i+1}
$$

are all torsion modules with $z^{-1}$ as annihilators, that is, for each $j$ and for each $[u] \in \mathscr{D}_{i} z^{-1}[u]=0$. Next we shall show that the sequence of quotient modules $\left\{\mathscr{D}_{i}\right\}$ is isomorphic to a chain $\left\{\boldsymbol{S}_{i}\right\}$ of (finite dimensional) $K$-linear subspaces of $U$, that is, each $\mathscr{D}_{i}$ is isomorphic to a subspace $S_{i} \subset U$ and

$$
\text { (6.7) } \quad 0=S_{k_{\Delta}-1} \subset S_{k_{\Delta}} \subset S_{k_{\Delta}+1} \subset \cdots \subset S_{j} \subset \cdots \subset U .
$$

Indeed, each element in $\mathscr{D}_{i}$ is an equivalence class $[u]$ of elements in $\Delta_{i}$. A representative $u \in[u]$ can be expressed as $u=\sum_{k=j}^{\infty} u_{k} z^{-k}$. If $u^{\prime}=\sum_{k=j}^{\infty} u_{k}^{\prime} z^{-k}$ and $u^{\prime \prime}=\sum_{k=j}^{\infty} u_{k}^{\prime \prime} z^{-k}$ are any two elements in the same equivalence class [u] then, since $u^{\prime}-u^{\prime \prime} \in \Delta_{j+1}$, it follows that $u_{i}^{\prime}=u_{i}^{\prime \prime}$. Thus, with each equivalence class [u] is associated a unique leading 
coefficient $u_{i}$ (of $z^{-j}$ ). We can now define the map $\gamma_{i}: \mathscr{D}_{i} \rightarrow U:[u] \mapsto u_{j}$. Naturally the map $\gamma_{i}$ is $K$-linear since $\gamma_{j}\left([u]+\left[u^{\prime}\right]\right)=\gamma_{i}\left(\left[u+u^{\prime}\right]\right)=u_{i}+u_{i}^{\prime}$ and $\gamma_{i}(\alpha[u])=\gamma_{j}([\alpha u])=\alpha u_{j}$. It is also clear that $\gamma_{j}$ is injective, since ker $\gamma_{j}=\Delta_{j+1}=$ [0]. Now, for each integer $j$ we define $S_{i}:=\operatorname{Im}\left(\gamma_{i}\right)$. Clearly $S_{i}$ is then $K$-linearly isomorphic to $\mathscr{D}_{i}$ and $S_{i} \subset S_{j+1}$ with $S_{k_{\Delta}-j-1}=0$ for all $j \geqq 0$. Also, by the finite dimensionality of $U$, there exists an integer $k^{\Delta^{2}}\left(\geqq k_{\Delta}\right)$ such that $S_{k^{\Delta}-1} \neq S_{k^{\Delta}}$ and $S_{k^{\Delta}+j}=S_{k^{\Delta}}$ for all $j \geqq 0$. We call the chain $\left\{S_{i}\right\}$ the orderchain of $\Delta$, and the sequence of integers $\left\{\mu_{i}\right\}, \mu_{i}:=\operatorname{dim} S_{j}$, we call the order list of $\Delta$ In the special case when $\Delta=\operatorname{ker} \pi^{-} \bar{f}$ where $\bar{f}$ is a linear i/o map, we refer to the order chain and the order list of $\Delta$, respectively, also as the latency chain and latency list of $\bar{f}$.

It is interesting to observe that the integer $k^{\Delta}$ is also the least integer satisfying the condition that $z^{-1} \Delta_{j}=\Delta_{j+1}$ for all $j \geqq k^{\Delta}$. Indeed, we have seen that $z^{-1} / j_{j} \subset \Delta_{j+1}$ for all $\mathrm{j}$. To see that $z^{-1} \Delta_{j} \supset \Delta_{j+1}$ if and only if $j \geqq k^{\Delta}$, let $u=\sum_{k=j+1}^{\infty} u_{k j} z^{-k} \in \Delta_{j+1}$ be any element. Then we can write $u=z^{-1} u^{\prime}$ where $u^{\prime}=\sum_{k=j}^{\infty} u_{k+1} z^{-k-j+1} \epsilon z^{-i} \Omega^{-} U$, and clearly $u \in z^{-1} \Delta_{j}$ if and only if $u^{\prime} \in \Delta_{i}$, This can hold for every $u \in \Delta_{i+1}$ only if $S_{i+1}=S_{i}$, whence the necessity that $j \geqq k^{\Delta}$. The sufficiency of the condition is an immediate consequence of Theorem 6.11 below.

Next we have the following useful result.

Lemma. 6.8. Let $\Delta \subset \Lambda U$ be a finitely generated $\Omega^{-} K$-submodule with order chain $\left\{S_{i}\right\}$ and order list $\left\{\mu_{i}\right\}$. Then $\operatorname{dim} S_{k^{\Delta}}=\operatorname{rank} \Delta$.

Proof. Let rank $\Delta=\mu$, let $d_{1}, \cdots, d_{\mu}$ be a basis of $\Delta$ and define $\mathscr{R}:=\operatorname{span}_{\Lambda K}\left\{d_{1}, \cdots, d_{\mu}\right\}$. It is easily seen that $\mathscr{R}$ is the smallest $\Lambda K$-linear space containing $\Delta$ and $\operatorname{dim}_{\Lambda K} \mathscr{R}=$ rank $\Delta$. The $\Lambda K$-linear space $\mathscr{R}$ has a proper basis and (by Corollary 4.5 ) $\operatorname{dim}_{\Lambda K} \mathscr{R}=\operatorname{dim}_{K} \hat{R}$. But clearly $\hat{\mathscr{R}}=S_{k^{2}}$ and the proof is complete.

Let $\left\{S_{i}\right\}$ and $\left\{S_{i}^{\prime}\right\}$ be the order chains and $\left\{\mu_{i}\right\}$ and $\left\{\mu_{i}^{\prime}\right\}$ the order lists, respectively of submodules $\Delta$ and $\Delta^{\prime}$ of $\Lambda U$. We shall say that $\left\{S_{i}^{\prime}\right\}$ is a subchain of $\left\{S_{i}\right\}$, denoted $\left\{S_{i}^{\prime}\right\} \subset\left\{S_{i}\right\}$ if, for all $j, S_{i}^{\prime} \subset S_{j}$. Similarly we say that the list $\left\{\mu_{i}^{\prime}\right\}$ is smaller than the list $\left(\mu_{i}\right\}$, denoted $\left\{\mu_{i}^{\prime}\right\} \leqq\left\{\mu_{i}\right\}$ if $\mu_{i}^{\prime} \leqq \mu_{i}$ for all integers $j$. As an immediate consequence of the definition we have the following,

Proposition. 6.9. Let $\Delta, \Delta^{\prime} \subset \Lambda U$ be $\Omega^{-} K$-submodules with order chains $\left\{S_{i}\right\}$ and $\left\{S_{i}^{\prime}\right\}$ and order lists $\left\{\mu_{i}\right\}$ and $\left\{\mu_{i}^{\prime}\right\}$, respectively. If $\Delta^{\prime} \subset \Delta$ then $\left\{S_{i}^{\prime}\right\} \subset\left\{S_{i}\right\}$ and $\left\{\mu_{i}^{\prime}\right\} \leqq\left\{\mu_{i}\right\}$.

Let $\Delta \subset \Lambda U$ be a finitely generated $\Omega^{-} K$-submodule. A set of elements $d_{1}, \cdots, d_{k} \in \Delta$ is called properly free if the elements are properly independent as elements of $\Lambda U$ (regarded as a $\Lambda K$-linear space), that is, if the leading coefficients $\hat{d}_{1}, \cdots, \hat{d}_{k}$ are $K$-linearly independent. It is then clear that if $d_{1}, \cdots, d_{k}$ are properly free they are also free (i.e. independent over the ring $\Omega^{-} K$ ).

Definition. 6.10. Let $\Delta \subset \Lambda U$ be a finitely generated $\Omega^{-} K$-submodule. A basis $d_{1}, \cdots, d_{\mu}$ of $\Delta$ is called proper if $d_{1}, \cdots, d_{\mu}$ are properly free. The basis will be called ordered if ord $d_{i+1} \geqq \operatorname{ord} d_{i}$ for all $i=1, \cdots, \mu-1$.

Theorem. 6.11. Let $\Delta \subset \Lambda U$ be an $\Omega^{-} K$-submodule or rank $\mu$ and of order $k_{\Delta}$, with order chain $\left\{S_{i}\right\}$ and order list $\left\{\mu_{i}\right\}$. Then (i) there exists an ordered proper basis for $\Delta$. (ii) If $d_{1}, \cdots, d_{\mu}$ is any ordered proper basis for $\Delta$, then the following conditions are satisfied:

(6.12) ord $d_{i}=i \quad$ for $\mu_{i-1}<j \leqq \mu_{i}$ and $i=k_{\Delta}, k_{\Delta+1}, \cdots$

(6.13) For each $j=1, \cdots, \mu$, the set $\hat{d}_{1}, \cdots, \hat{d}_{j} \in S_{i}$, where $i$ is the least integer such that $j \leqq \mu_{i}$.

Proof. (i) We shall construct an ordered proper basis for $\Delta$ which, in particular, satisfies (6.12) and (6.13). Consider the sequence $\left\{\mathscr{D}_{i}\right\}$ of quotient modules $\mathscr{D}_{j}$ defined by (6.6), of which $\mathscr{D}_{k_{\Delta}}$ is the first nonzero one. Choose any equivalence class $0 \neq\left[d_{1}\right] \epsilon \mathscr{D}_{k_{\Delta}}$ and let $d_{1} \in \Delta$ be any representative of $\left[d_{1}\right]$. Then ord $d_{1}=k_{\Delta}$ and $d_{1}$ is clearly properly free. We proceed stepwise and assume that for $j>0, d_{1}, \cdots, d_{j}$ are properly free elements of $\Delta$ satisfying (6.12) and (6.13). If $j<\mu$, let $k$ denote the least integer such that $j<\mu_{k}$. Then $\hat{d}_{1}, \cdots, \hat{d}_{i} \in S_{k}$ are $K$-linearly independent, but they do not span $S_{k}, \operatorname{since} \operatorname{dim} S_{k}=\mu_{k}$. Thus, there exists an element $\left[d_{i+1}\right] \in \mathscr{D}_{k}$ such that for any representative $d_{j+1} \in\left[d_{j+1}\right]$, the set $\hat{d}_{1}, \cdots, \hat{d}_{j}, \hat{d}_{j+1} \in S_{k}$ are $K$-linearly independent and hence the set $d_{1}, \cdots, d_{i+1}$ is properly free. Clearly (6.13) is satisfied, and since ord $d_{j+1}=k$ so is also (6.12). By Lemma 6.8, $\operatorname{dim} S_{k^{\Delta}}=\operatorname{rank} \Delta=$ $\mu$, so that we finally obtain an ordered, properly free set of elements $d_{1}, \cdots, d_{\mu} \in \Delta$ satisfying (6.12) and (6.13). Let $\Delta^{\prime}$ denote the $\Omega^{-} K$-submodule of $\Lambda U$ generated by $d_{1}, \cdots, d_{\mu}$. It remains to be shown that $\Delta^{\prime}=\Delta$. Obviously $\Delta^{\prime} \subset \Delta$

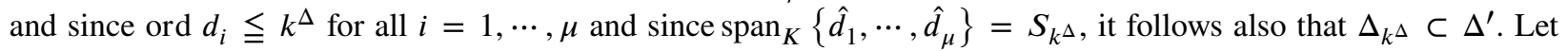
$u \in \Delta$ be any element and let ord $u=j$. Then $\hat{u} \in S_{i}$ whence there are elements $\alpha_{1}, \cdots, \alpha_{\mu_{i}} \in \Omega^{-} K$ such that 
$\sum_{k=1}^{\mu_{i}} \hat{\alpha}_{k} \hat{d}_{k}=\hat{u}$ and ord $\left(u-\sum_{k=1}^{\mu_{i}} \alpha_{k} d_{k}\right)>j$. Proceeding stepwise the same way, we conclude that there are elements $\alpha_{1}, \cdots, \alpha_{\mu} \in \Omega^{-} K$ such that $u=\sum_{i=1}^{\mu} \alpha_{i} d_{i}+u^{\prime}$, with ord $u^{\prime} \geqq k^{\Delta}$. Clearly, $\sum_{i=1}^{\mu} \alpha_{i} d_{i} \in \Delta^{\prime}$, and since $u^{\prime} \in \Delta_{k^{2}} \subset \Delta^{\prime}$, it follows also that $u \in \Delta^{\prime}$ and the proof of (i) is complete. To see that (ii) holds, it suffices to observe that for each integer $j$, every ordered proper basis $d_{1}, \cdots, d_{\mu}$ of $\Delta$ has precisely $\mu_{i}$ elements whose order is less than or equal to $j$ and $\operatorname{span}_{K}\left\{\hat{d}_{1}, \cdots, \hat{d}_{\mu}\right\}=S_{j}$.

The following immediate corollary to Theorem 6.11 gives a sharp insight to the relation between ordered proper bases of $\Omega^{-} K$-modules and their order chain

Corollary. 6.14. Let $\Delta \subset \Lambda U$ be an $\Omega^{-} K$-submodule of rank $\mu$ with order chain $\left\{S_{i}\right\}$ and order list $\left\{\mu_{i}\right\}$. Then $d_{1}, \cdots, d_{\mu}$ is an ordered proper basis of $\Delta$ if and only if for each $j, \hat{d}_{1}, \cdots, \hat{d}_{\mu_{i}}$ is a basis for $S_{i}$.

We now return to questions connected with our primary objective of studying causal factorization and feedback. First we have some preliminary facts

Lemma. 6.15. Let $U$ be an $m$-dimensional K-linear space and let $\bar{f}: \Lambda U \rightarrow \Lambda Y$ be a MK-linear map. For each integer $j$ let $\Delta_{j}(\bar{f})$ the $\Omega^{-} K$-subodule of $\Lambda U$ defined by $\Delta_{j}(\bar{f}):=\operatorname{ker} \pi^{-} \bar{f} \cap z^{-j} \Omega^{-} U$. Then rank $\Delta_{j}(\bar{f})=m$.

Proof. First note that since $\Delta_{j}(\bar{f}) \subset z^{-j} \Omega^{-} U$, rank $\Delta_{j}(\bar{f}) \leqq m$, with equality obviously holding when $\bar{f}=0$, since then ker $\pi^{-} \bar{f}=\Lambda U$. Assume now that $\bar{f} \neq 0$, define $t:=\max \{j-$ ord $\bar{f},-$ ord $\bar{f}\}$ and let $u \in z^{-1} \Omega^{-} U_{-}$be any element. Then ord $\bar{f} u \geqq \operatorname{ord} \bar{f}+$ ord $u \geqq$ ord $\bar{f}+t \geqq \max \{j, 0\}$ and $u \in \Delta_{j}(\bar{f})$. Hence $z^{-1} \Omega^{-} U \subset \Delta_{i}(\bar{f})$ so that $\operatorname{rank} \Delta_{j}(\bar{f}) \geqq m$ and the proof is complete.

Proposition. 6.16. Let $U$ be an m-dimensional K-linear space and let $\bar{f}: \Lambda U \rightarrow \Lambda Y$ be a $\Lambda K$-linear map. Then the following are equivalent

(i) $\bar{f}$ is injective.

(ii) $\operatorname{ker} \pi^{-1} \bar{f}$ is finitely generated.

(iii) $\operatorname{rank} \operatorname{ker} \pi^{-} \bar{f}=m$.

Proof. That (ii) and (iii) are equivalent follows immediately from Lemma 6.15 and the fact that if ker $\pi^{-1} \bar{f}$ is finitely generated it is of finite order, say $t$, so that ker $\pi^{-} \bar{f}=\Delta_{t}(\bar{f})$. To see that (ii) implies (i), recall that ker $\bar{f} \subset \operatorname{ker} \pi^{-} \bar{f}$ so that if ker $\bar{f} \neq 0$ then ker $\pi^{-} \bar{f}$ is not of finite order and hence is not finitely generated. It remains to be shown that (i) implies (ii). Assume that (i) holds, let $y_{1}, \cdots, y_{m}$ be a normalized proper basis for $\operatorname{Im} \bar{f} \subset \Lambda Y$ and let $u_{1}, \cdots, u_{m}$ be the (unique) elements of $\Lambda U$ satisfying $\bar{f}\left(u_{i}\right)=y_{i}, i=1, \cdots, m$. The proof will be complete upon showing that ker $\pi^{-} \bar{f}$ is of finite order and, in fact, we claim that ker $\pi^{-} \bar{f} \subset z^{-t} \Omega^{-} U$ where $t:=\min \left\{\operatorname{ord} u_{i} \mid i=1, \cdots, m\right\}$. Indeed, if $u \in \operatorname{ker} \pi^{-} \bar{f}$, then $\bar{f}(u) \in \Omega^{-} Y$ and there are elements $\alpha_{1}, \cdots, \alpha_{m} \in \Omega^{-} K$ such that $\bar{f}(u)=\sum_{i=1}^{m^{\prime}} \alpha_{i} y_{i}=$ $\sum_{i=1}^{m} \alpha_{i} \bar{f}\left(u_{i}\right)=\bar{f}\left(\sum_{i=1}^{m} \alpha_{i} u_{i}\right)$ whence $u=\sum_{i=1}^{m} \alpha_{i} u_{i}$ so that ord $u \geqq t$.

In view of Proposition 6.16, it follows that the latency kernel of a given linear i/o map $\bar{f}$ is finitely generated if and only if $\bar{f}$ is injective, the case which receives, of course, most of our attention. Before proceeding further, a remark on the noninjective case is in order

Remark. 6.17. It is readily noted that if $\bar{f}: \Lambda U \rightarrow \Lambda Y$ is a $\Lambda K$-linear map, then ker $\pi^{-} \bar{f}$ can (always) be written as

$$
\operatorname{ker} \pi^{-} \bar{f}=\operatorname{ker} \bar{f}+\mathscr{R}
$$

where $\mathscr{R}$ is a finitely generated full $\Omega^{-} K$-submodule of $\Lambda U$. However, in the above representation, $\mathscr{R}$ is nonunique except in the special case when $\bar{f}$ is injective and ker $\bar{f}=0$. If $\bar{f}_{1}$ and $\bar{f}_{2}$ are two $\Lambda K$-linear maps then ker $\pi^{-} \bar{f}_{1} \subset$ ker $\pi^{-} \bar{f}_{2}$ if and only if ker $\bar{f}_{1}+\mathscr{R}_{1} \subset \operatorname{ker} \bar{f}_{2}+\mathscr{R}_{2}$. While this condition necessarily implies ker $\bar{f}_{1} \subset \operatorname{ker} \bar{f}_{2}$, it cannot be claimed, except in the injective case, that $\mathscr{R}_{1} \subset \mathscr{R}_{2}$. Hence, for computational purposes it is convenient in the noninjective case to resort to the fact that $\operatorname{ker} \pi^{-} \bar{f}_{1} \subset \operatorname{ker} \pi^{-} \bar{f}_{2}$ if and only if $\Delta_{i}\left(\bar{f}_{1}\right) \subset \Delta_{j}\left(\bar{f}_{2}\right)$ for all $j$, where $\Delta_{j}\left(\bar{f}_{i}\right)$ is as defined in Lemma 6.15 However, $\Delta_{j}\left(\bar{f}_{1}\right) \subset \Delta_{j}\left(\bar{f}_{2}\right)$ for all $j$ if and only if $\Delta_{i}\left(\bar{f}_{1}\right) \subset \Delta_{i}\left(\bar{f}_{2}\right)$ for any $j \leqq \min \left\{\right.$ ord $\mathscr{R}_{1}$, ord $\left.\mathscr{R}_{2}\right\}$ where $\mathscr{R}_{i}, i=1,2$, are any submodules in the corresponding representations of ker $\pi^{-} \bar{f}_{i}$. By Lemma 6.15 both $\Delta_{j}\left(\bar{f}_{1}\right)$ and $\Delta_{j}\left(\bar{f}_{2}\right)$ are full finitely generated $\Omega^{-} K$-submodules of $\Lambda U$ so that the situation is thus similar to that in the injective case.

Let $\bar{f}: \Lambda U \rightarrow \Lambda Y$ be an injective extended linear i/o map and let $\Delta=\operatorname{ker} \pi^{-} \bar{f}$. Then $\Delta=D \Omega^{-} U$ is a full, finitely generated $\Omega^{-} K$-submodule of $\Lambda U$ and the columns $d_{1}, \cdots, d_{m}$ of the generating matrix $D$ form a basis of $\Delta$. We shall next establish certain properties of possible selections of the matrix $D$. 
Proposition. 6.18. Let $\bar{f}: \Lambda U \rightarrow \Lambda Y$ be an injective extended linear i/o map. Write ker $\pi^{-} \bar{f}=D \Omega^{-} U$. Then $D^{-1}$ exists and is strictly causal; i.e., the elements of $D^{-1}$ are in $z^{-1} \Omega^{-} K$.

Proof. The existence of $D^{-1}$ follows immediately from Proposition 6.16. From the strict causality of $\bar{f}$ it follows that $z \Omega^{-} U \subset$ ker $\pi^{-} \bar{f}$, whence by Theorem 6.2 there exists a causal matrix $R$ such that $z I=D R$. Thus $D^{-1}=z^{-1} R$ and $z^{-1} R$ is clearly strictly causal.

Let $\Delta \subset \Lambda U$ be a full finitely generated $\Omega^{-} K$-submodule and write $\Delta=D \Omega^{-} U$. We call the columns $d_{1}, \cdots, d_{m}$ of $D$ a polynomial be : is of $\Delta$ if the matrix $D$ is a polynomial matrix, i.e., with elements in $\Omega^{+} K$. We call the basis a strictly polynomial basis if its elements are strict polynomials, i.e., with elements in $z \Omega^{+} K$. If in addition $D$ is a proper basis we call it a proper polynomial basis, respectively, proper strictly polynomial basis for $\Delta$.

Theorem. 6.19. Let $\bar{f}: \Lambda U \rightarrow \Lambda Y$ be an injective extended linear i/o map. Then $\mathrm{ker} \pi^{-} \bar{f}$ has a proper strictly polynomial basis.

Proof. Let $\tilde{d}_{1}, \cdots, \tilde{d}_{m}$ be a proper basis for ker $\pi^{-} \bar{f}$ and for each $i$ write $\tilde{d}_{i}=\Sigma d_{i j} \cdot z^{-i}=d_{i}+d_{i}^{-}$, where $d_{i}=$ $\sum_{i<0} d_{i j} z^{-j} \in z \Omega^{+} U$ and $d_{i}^{-}=\sum_{i \geq 0} d_{i j} z^{-j} \in \Omega^{-} U$. Then $z d_{i}^{-} \in z \Omega^{-} U \subset \operatorname{ker} \pi^{-} \bar{f}$, the inclusion following from the strict causality of $\bar{f}$. Thus there are elements $\alpha_{i j} \in \Omega^{-} K, j=1, \cdots, m$, so that $z d_{i}^{-}=\sum_{i=1}^{m} \alpha_{i j} \tilde{d}_{i}$ Defining the matrices $D:=\left[d_{1}, \cdots, d_{m}\right], \tilde{D}:=\left[\tilde{d}_{1}, \cdots, \tilde{d}_{m}\right]$ and $A:=\left[\alpha_{i j}\right]$ we can thus write $\tilde{D}=D+z^{-1} \tilde{D} A$, or alternatively, $D=$ $\tilde{D}\left(I-z^{-1} A\right)$. since $A$ is causal by definition of the $\alpha_{i j}$ it follows that $\left(I-z^{-1} A\right)$ is a bicausal matrix. Consequently, by Corollary 6.3, we have ker $\pi^{-} \bar{f}=\tilde{D} \Omega^{-} U=D \Omega^{-} U$ so that the columns $d_{1}, \cdots, d_{m}$ of $D$ also form a proper basis for ker $\pi^{-} \bar{f}$. That this basis is strictly polynomial follows directly from the definition of the $d_{i}$.

For an injective extended linear i/o map $\bar{f}$ it is convenient to define a set of nonnegative integers, called latency indices, which are associated in one-one correspondence with the latency list of $\bar{f}$. We proceed as follows. Let $d_{1}, \cdots, d_{m}$ be an ordered proper basis for ker $\pi^{-} \bar{f}$. Then, as we have seen, for each $i=1, \cdots, m$ ord $d_{i} \leqq-1$. We define the latency indices $\left\{v_{1}, \cdots, v_{m}\right\}$ of $\bar{f}$ by $v_{i}:=-$ ord $d_{i}-1$. The relation of the latency indicates with the latency list is clearly established by Corollary 6.14, and if $\left\{\mu_{i}\right\}$ is the latency list of $\bar{f}$ then we have

$$
v_{i}=-j-1 \text { for } \mu_{j-1}<i \leqq \mu_{i}, \quad j=k_{\Delta}, k_{\Delta}+1, \cdots
$$

where $k_{\Delta}=\operatorname{ord} \operatorname{ker} \pi^{-} \bar{f}$. Clearly $v_{i} \geqq 0$ for all $i=1, \cdots, m$, and $\bar{f}$ is nonlatent if and only if all its latency indices are zero.

We conclude this section with the discussion of certain invariance properties of the latency indices. We have seen previously that if $\bar{f}_{1}: \Lambda U \rightarrow \Lambda Y$ and $\bar{f}_{2}: \Lambda U \rightarrow \Lambda Y$ are two extended linear i/o maps and if $\bar{l}_{\mathrm{po}}: \Lambda Y \rightarrow \Lambda Y$ is a $\Lambda K$-linear bicausal isomorphism such that $\bar{f}_{2}=\bar{l}_{\mathrm{po}} \cdot \bar{f}_{1}$, then $\bar{f}_{1}$ and $\bar{f}_{2}$ have the same latency kernels; i.e., ker $\pi^{-} \bar{f}_{1}=\operatorname{ker} \pi^{-} \bar{f}_{2}$. If there exist both a bicausal postcompensator as above and a $\Lambda K$-linear bicausal precompensator $\bar{l}_{\mathrm{pr}}: \Lambda U \rightarrow \Lambda U$ such that $\bar{f}_{2}=\bar{l}_{\mathrm{po}} \cdot \bar{f}_{1} \cdot \bar{l}_{\mathrm{pr}}$, then $\operatorname{ker} \pi^{-} \bar{f}_{2}=\operatorname{ker} \pi^{-} \bar{f}_{1} \cdot \overline{1}_{\mathrm{pr}}$, and since $u \in \operatorname{ker} \pi^{-} \bar{f}_{1} \cdot \bar{l}_{\mathrm{pr}}$ if and only if $\bar{l}_{\mathrm{pr}} u \in \operatorname{ker} \pi^{-} \bar{f}_{1}$, it follows that $\bar{l}_{\mathrm{pr}} \operatorname{ker} \pi^{-1} \bar{f}_{2}=\operatorname{ker} \pi^{-} \bar{f}_{1}$. since the map $\bar{l}_{\mathrm{pr}}$ is, in particular, also an $\Omega^{-} K$-homorphism (which we denote $l_{\mathrm{pr}}$ ) we interpret it as an order preserving $\Omega^{-} K$-isomorphism $l_{\mathrm{pr}}: \operatorname{ker} \pi^{-} \bar{f}_{2} \rightarrow \operatorname{ker} \pi^{-} \bar{f}_{1}$. Suppose, conversely, that there exists an order preserving $\Omega^{-} K$-isomorphism $l_{\mathrm{pr}}$ as above. Fix an integer $j$ and define (as in Lemma 6.15) $\Delta_{j}\left(\bar{f}_{2}\right) \subset \operatorname{ker} \pi^{-} \bar{f}_{2}$. Then, by the same lemma, $\Delta_{j}\left(\bar{f}_{2}\right)$ is a full finitely generated $\Omega^{-} K$ submodule of $\Lambda U$, and if $d_{1}, \cdots, d_{m}$ is a proper basis for $\Delta_{i}\left(\bar{f}_{2}\right)$, it is clearly also a basis for $\Lambda U$. Let $\bar{l}_{\mathrm{pr}}: \Lambda U \rightarrow \Lambda U$ be the (unique) $\Lambda K$-linear map whose action on the $d_{i}^{\prime} \mathrm{s}$ is that of $l_{\mathrm{pr}}$. Then, $\bar{l}_{\mathrm{pr}}$ is order preserving and thus a bicausal isomorphism $\Lambda U \rightarrow \Lambda U$ Moreover, since $\bar{l}_{\mathrm{pr}} u=l_{\mathrm{p} t} u$ for all elements $u \in \operatorname{ker} \pi^{-} \bar{f}_{2}$, it follows that $\bar{l}_{\mathrm{pr}} \operatorname{ker} \pi^{-} \bar{f}_{2}=\operatorname{ker} \pi^{-} \bar{f}_{1}$ whence ker $\pi^{-} \bar{f}_{2}=\operatorname{ker} \pi^{-} \bar{f}_{1} \cdot \bar{l}_{\mathrm{pr}}$. Applying now Corollary 5.7 to the above kernel equality, we conclude that there exists a bicausal $\Lambda K$-linear postcompensator $\bar{l}_{\mathrm{po}}: \Lambda Y \rightarrow \Lambda Y$ such that $\bar{f}_{2}=\bar{l}_{\mathrm{po}} \bar{f}_{1} \bar{l}_{\mathrm{pr}}$. We have just proved the following.

Theorem. 6.21. Let $\bar{f}_{1}, \bar{f}_{2}: \Lambda U \rightarrow \Lambda Y$ be two extended linear i/o maps with $U$ and $Y$ finite dimensional $K$-linear spaces. There exist bicausal $\Lambda K$-linear compensators $\bar{l}_{\mathrm{pr}}: \Lambda U \rightarrow \Lambda U$ and $\bar{l}_{\mathrm{po}}: \Lambda Y \rightarrow \Lambda Y$ such that $\bar{f}_{2}=\bar{l}_{\mathrm{po}} \cdot \bar{f}_{1} \cdot \bar{l}_{\mathrm{pr}}$ if and only if there exists an order preserving $\Omega^{-} K$-isomorphism $l_{\mathrm{pr}}: \operatorname{ker} \pi^{-} \bar{f}_{2} \rightarrow \operatorname{ker} \pi^{-} \bar{f}_{1}$.

We now restrict Theorem 6.21 to the injective case to obtain the following invariance characterization of the latency indices

Corollary. 6.22. Let $\bar{f}_{1}, \bar{f}_{2}: \Lambda U \rightarrow \Lambda Y$ be two injective extended linear i/o maps with $U$ and $Y$ finite dimensional $K$-linear spaces. There exist bicausal $\Lambda K$-linear compensators $\bar{l}_{\mathrm{pr}: \Lambda U} \rightarrow \Lambda U$ and $\bar{l}_{\mathrm{po}}: \Lambda Y \rightarrow \Lambda Y$ such that $\bar{f}_{2}=\bar{l}_{\mathrm{po}} \cdot \bar{f}_{1} \cdot \bar{l}_{\mathrm{pr}}$ if and only if $\bar{f}_{1}$ and $\bar{f}_{2}$ have the same latency indices. 
Proof. By the injectivity of $\bar{f}_{1}$ and $\bar{f}_{2}$, both $\Delta_{1}=\operatorname{ker} \pi^{-} \bar{f}_{1}$ and $\Delta_{2}=\operatorname{ker} \pi^{-} \bar{f}_{2}$ are of rank $m$, where $m=\operatorname{dim} U$, and in view of Theorem 6.21 it needs only to be shown that $\Delta_{1}$ and $\Delta_{2}$ have the same latency indices (or latency lists) if and only if there exists an order preserving $\Omega^{-} K$-isomorphism $l_{\mathrm{pr}}: \Delta_{2} \rightarrow \Delta_{1}$. Let $d_{11}, \cdots, d_{1 m}$ and $d_{21}, \cdots, d_{2 m}$ be ordered proper bases for $\Delta_{1}$ and $\Delta_{2}$, respectively, and let $D_{1}$ and $D_{2}$ be the corresponding matrices. Then an order preserving isomorphism $l_{\mathrm{pr}}: \Delta_{2} \rightarrow \Delta_{1}$ exists if and only if the matrix $D_{1} D_{2}^{-1}$ is bicausal which is easily seen to be the case if and only if ord $d_{1 j}=$ ord $d_{2 j}$ for all $j=1, \cdots, m$. Employing Corollary 6.14 completes the proof.

Theorem 6.21 and Corollary 6.22 could, of course, have been stated for any $\Lambda K$-linear maps and not only strictly causal ones. The proofs did in no way depend on the causality properties of the maps involved. Also, Corollary 6.22 could have been obtained as an application of the existence of, so called, Smith canonical forms for matrices over Euclidean rings (see, e.g., MacDuffee [1934]).

\section{PRECOMPENSATION AND FEEDBACK.}

Let $\bar{f}: \Lambda U \rightarrow \Lambda Y$ be an extended linear i/o map and let $\bar{l}: \Lambda U \rightarrow \Lambda U$ be a $\Lambda K$-linear bicausal precompensator. Write $\bar{l}^{-1}=L+h$ where $L: \Lambda U \rightarrow \Lambda U$ is static and $\bar{h}: \Lambda U \rightarrow \Lambda U$ is strictly causal. We have seen in $\$ 5$ that $\bar{l}$ can be realized by a static precompensator (i.e., coordinate change in the input value space and output feedback around $\tilde{f}_{-}$(i.e., $\bar{h}=\bar{g} \cdot \bar{f}$ for causal $\Lambda K$-linear map $\bar{g}: \Lambda Y \rightarrow \Lambda U$ ) if and only if ker $\pi^{-} \bar{f} \subset \operatorname{ker} \pi^{-} \bar{h}$ (see Theorem 5.2). When $\bar{f}$ is a nonlatent map, feedback realization as above is thus possible for every bicausal map $\bar{l}$. In general, however, feedback realization is not possible for every precompensator $\bar{l}$. We shall say that $\bar{l}$ has $a(\bar{v}, \bar{g})$ representation if it can be expressed as $\bar{l}=\bar{l}_{(\bar{v}, \bar{\varepsilon})}=(I+\bar{g} \bar{f})^{-1} \bar{v}$ where $\bar{v}: \Lambda U \rightarrow \Lambda U$ is a bicausal isomorphism and $\bar{g}: \Lambda Y \rightarrow \Lambda U$ is a causal $\Lambda K$-linear map. We call the map $\bar{v}$ in the above representation the precompensator remainder of the representation. The precompensator $\bar{l}$ can thus be realized as feedback whenever $\bar{l}$ has a $(\bar{v}, \bar{g})$ representation with $\bar{v}=V$, a static map.

In general, the precompensator remainder $\bar{v}$ is dynamic and can be represented as $\bar{v}=V+\bar{v}_{c}$ where $V$ is the static part of $\bar{v}$ and $\bar{v}_{c}: \Lambda U \rightarrow \Lambda U$ is strictly causal, i.e., an extended linear i/o map. We recall (see, in particular, Hautus and Heymann [1978]) that the dynamic characteristics of $\bar{v}_{c}$ are determined by ker $\pi^{+} \bar{v}_{c} \cdot j^{+}$which is an $\Omega^{+} K$-submodule of $\Omega^{+} U$ and can be represented by

(7.1) $\operatorname{ker} \pi^{+} \bar{v}_{c} \cdot j^{+}=\operatorname{ker} \pi^{+} \bar{v} \cdot j^{+}=D \Omega^{+} U$,

where $D$ is a polynomial matrix whose columns form a basis for ker $\pi^{+} \bar{v} \cdot j^{+}$. The degree $n$ of the determinant of $D$ (when $D$ is nonsingular) is the dimension of the minimal state space realizing $\bar{v}_{c}$. More specifically, if $D$ in (7.1) is selected to be proper, i.e., the columns of $D$ are properly free (in the sense that the leading coefficient vectors are $K$ -linearly independent just as in 84 above), then the column degrees $\sigma_{i}, i=1, \cdots, m$ are the reachability indices of $\bar{v}_{c}$ and their sum is $\sum_{i=1}^{m} \sigma_{i}=n=\operatorname{deg} \cdot \operatorname{det} D$.

It is of interest in selecting a $(\bar{v}, \bar{g})$ pair representing a given precompensator $\bar{l}$ to choose the representation in such a way that the precompensator remainder $\bar{v}$ has least dynamic order, i.e., is realizable by a state space of least possible dimension. In this way the precompensator is realized "as much as possible" by feedback. The following theorem provides a bound on the dynamic order of the precompensator remainder $\bar{v}$ which need not be exceeded in the realization of any bicausal precompensator $\bar{l}$, and which is dependent only on the dynamic properties (latency) of the i/o map $\bar{f}$ under consideration.

Theorem. 7.2. Let $\bar{f}: \Lambda U \rightarrow \Lambda Y$ be an injective extended linear i/o map with latency indices $v_{1} \geqq \cdots \geqq v_{m}$. Let $\bar{l}: \Lambda U \rightarrow \Lambda U$ be a bicausal $\Lambda K$-linear map. There exists a $(\bar{v}, \bar{g})$ representation for $\bar{I}$ such that the precompensator remainder $\bar{v}$ has (ordered) reachability indices $\sigma_{1} \geqq \cdots \geqq \sigma_{m}$ satisfying $\sigma_{i} \leqq v_{i}, i=1, \cdots, m$.

Remark. 7.3. It is interesting to observe that Theorem 7.2 explicitly implies what we have seen previously, namely, that if $\bar{f}$ is a nonlatent i/o map, then every bicausal $\bar{l}$ can be realized as output feedback. Indeed, if $\bar{f}$ is nonlatent, its latency indices $v_{i}$ are all zero, whence by Theorem 7.2 there exists a pair $(\bar{v}, \bar{g})$ with $\bar{v}$ having reachability indices all zero, that is, with $\bar{v}$ static.

To prove Theorem 7.2 we shall need the following lemmas.

Lemma. 7.4. Let $U$ be a finite dimensional $K$-linear space and let $\bar{v}: \Lambda U \rightarrow \Lambda U$ be a bicausal $\Lambda K$-linear isomorphism. Then $\operatorname{ker} \pi^{+} \bar{v} \cdot j^{+}$and $\operatorname{ker} \pi^{+} \bar{v}^{-1} \cdot j^{+}$have the same lists of reachability indices.

Proof. By Hautus and Heymann [1978, Theorem 6.11] the lemma will be proved upon showing that there exists an order-preserving $\Omega^{+} K$-isomorphism $\operatorname{ker} \pi^{+} \bar{v} \cdot j^{+} \rightarrow \operatorname{ker} \pi^{+} \bar{v}^{-1} \cdot j^{+}$. We shall see that the map $\bar{v}$ itself, which is in particular also an order preserving $\Omega^{+} K$-isomorphism, satisfies the required properties. Indeed, let $\xi \in \operatorname{ket} \pi^{+} \bar{v} \cdot j^{+}$ 
be any element. Then $\bar{v} \cdot j^{+} \xi=\bar{v} \xi \in \Omega^{+} U$ and since also $\xi \in \Omega^{+} U$ we have $\xi=\bar{v}^{-1}(\bar{v} \xi)=\bar{v}^{-1} j^{+}(\bar{v} \xi) \in \Omega^{+} U$, whence $\bar{v} \xi \in \operatorname{ker} \pi^{+} \bar{v}^{-1} \cdot j^{+}$, completing the proof. $\square$

Let $\bar{f}: \Lambda U \rightarrow \Lambda Y$ be an injective extended linear i/o map and let $d_{1}, \cdots, d_{m}$ be a proper strictly polynomial basis for ker $\pi^{-} \bar{f}$ (see Theorem 6.19), and write ker $\pi^{-} \bar{f}=D \Omega^{-} U$ where $D=\left[d_{1}, \cdots, d_{m}\right]$. Then $z^{-1} D$ is also polynomial and the column degrees of $z^{-1} D$ are (by definition) the latency indices of $\bar{f}$. Below we shall not distinguish sharply between maps and their transfer functions. Let $\mathcal{S}^{-}: \Lambda U \rightarrow \Omega^{-} U: \Sigma u_{i} z^{-t} \mapsto \Sigma_{t \geq 0} u_{t} z^{-t}$ denote the causal truncation. Let $N: \Lambda U \rightarrow \Omega^{-} U$ be defined as the (unique) $\Lambda K$-linear map whose transfer function is given by

$$
\text { (7.5) } \quad N:=\mathcal{S}^{-}\left(\bar{l}^{-1} D\right) \text {, }
$$

and define the $\Lambda K$-linear maps

$$
\begin{aligned}
& \bar{\phi}: \Lambda U \rightarrow \Lambda U: u \mapsto N D^{-1} u, \\
& \text { (7.7) } \quad \bar{v}^{-1}:=\bar{l}^{-1}-\bar{\phi} .
\end{aligned}
$$

Lemma. 7.8. With $\bar{\phi}$ and $\bar{v}^{-1}$ as defined in (7.6) and (7.7) the following hold true:

(i) $\operatorname{ker} \pi^{-} \bar{f} \subset \operatorname{ker} \pi^{-} \bar{\phi}$.

(ii) $z^{-1} D \Omega^{+} U \subset \operatorname{ker} \pi^{+} \cdot \bar{v}^{-1} \cdot j^{+}$.

Proof. z(i) Let $u \in \operatorname{ker} \pi^{-} \bar{f}$. Then $u=D w$ for some $w \in \Omega^{-} U$ and we have $\bar{\phi} u=N D^{-1} u=N D^{-1} D w=N w \in$ $\Omega^{-} U$ since $N$ is a causal map, and hence $\pi^{-} \bar{\phi} u=0$ so that $u \in \operatorname{ker} \pi^{-} \bar{\phi}$. (ii) If $u \in z^{-1} D \Omega^{+} U$ then $u=z^{-1} D w$ for some $w \in \Omega^{+} U$, and we have, using the definitions of $\bar{v}^{-1}$ and of $\bar{\phi}, \bar{v}^{-1} j^{+} \underline{u}=\bar{v}^{-1} z^{-1} D w=\left(\bar{l}^{-1}-\bar{\phi}\right) z^{-1} D w=$ $z^{-1}\left(\bar{l}^{-1} D-N\right) w$. Now, in view of (7.5) the map $\left(\bar{l}^{-1} D-N\right)$ has a strictly polynomial transfer function so that $z^{-1}\left(\bar{l}^{-1} D-N\right)$ is polynomial. since also $w$ is polynomial it follows that $z^{-1}\left(\bar{l}^{-1} D-N\right) w \in \Omega^{+} U$, whence $u \in$ $\operatorname{ker} \pi^{+} \bar{v}^{-1} j^{+}$as claimed.

Proof of Theorem 7.2. If $\bar{l}$ is a bicausal precompensator for $\bar{f}$ and $(\bar{v}, \bar{g})$ is a representation of $\bar{l}$, then $\bar{l}=(I+\bar{g} \cdot \bar{f})^{-1} \bar{v}$, whence $\bar{l}^{-1}=\bar{v}^{-1}+\bar{v}^{-1} \cdot \bar{g} \cdot \bar{f}=\bar{v}^{-1}+\bar{\rho} \cdot \bar{f}$ where the map $\bar{\rho}=\bar{v}^{-1} \bar{g}$ is clearly also causal. By Lemma $7.4, \bar{v}$ and $\bar{v}^{-1}$ have the same reachability indices. Hence the theorem will be proved if we can show that $\bar{l}^{-1}$ can be represented as

$$
\bar{l}^{-1}=\bar{v}^{-1}+\bar{\phi}
$$

satisfying the following requirements: (a) $\bar{v}^{-1}: \Lambda U \rightarrow \Lambda U$ is a bicausal $\Lambda \mathrm{K}$-linear map such that its reachability indices $\sigma_{i}$ satisfy $\sigma_{i} \leqq v_{i}, i=1, \cdots, m$. (b) The $\Lambda K$-linear map $\bar{\phi}: \Lambda U \rightarrow \Lambda U$ is strictly causal and can be represented as $\bar{\phi}=\bar{\rho} \cdot \bar{f}$ for some causal $\Lambda K$-linear map $\bar{\rho}: \Lambda Y \rightarrow \Lambda U$. As we see below, the maps $\bar{\phi}$ and $\bar{v}^{-1}$ as defined in (7.6) and (7.7) satisfy the required conditions. Indeed, Lemma 7.8(i) combined with Theorem 5.2 implies that $\bar{\phi}=\bar{p} \cdot \bar{f}$ for some causal $\bar{\rho}$. since $\bar{f}$ is strictly causal by definition, it follows that so also is $\bar{\phi}$. Hence condition (b) above holds. To see that (a) is also satisfied note first that the difference between a bicausal $\Lambda K$-linear map and a strictly causal one is bicausal (see e.g. Corollary 2.11 ). Hence the map $\bar{v}^{-1}$ is bicausal. Now Lemma 7.8 (ii) implies the requirement on the reachability indices since, in particular, it implies that $\bar{v}^{-1}$ can be realized with state space $\Omega^{+} U / z^{-1} D \Omega^{+} U$ whose reachability indices are the column degrees of $z^{-1} D$. (The reader is referred to Hautus and Heymann [1978] for relevant details on the problem of realization.)

While Theorem 7.2 gives an upper bound on the required dynamic order of precompensator remainders, it has been, so far, seen only in the nonlatent case that this bound is tight. It is clear that in general, except in the case of nonlatent i/o maps, the maximal required order of precompensator remainders depends not only on the i/o map $\bar{f}$ but also on the specific precompensator $\bar{l}$ under consideration. It turns out that the bound of Theorem 7.2 is tight, however, in the following sense: There always exist bicausal isomorphisms $\bar{l}$ for which all precompensator remainders satisfy the condition that $n=\sum_{i=1}^{m} \sigma_{i} \geqq \sum_{i=1}^{m} v_{i}$, where $n$ is the minimal state space dimension and the $\sigma_{i}$ are reachability indices of the precompensator remainder, and the $v_{i}$ are the latency indices of the i/o, map $\bar{f}$.

Theorem. 7.9. Let $\bar{f}: \Lambda U \rightarrow \Lambda Y$ be an injective linear i/o map with latency indices $v_{1}, \cdots, v_{m}$. There exists a $\Lambda K$ -linear bicausal isomorphism $\bar{l}: \Lambda \dot{U} \rightarrow \Lambda U$ such that the following holds: If $(\bar{v}, \bar{g})$ is any representation of $\bar{l}$ and if $\sigma_{1}, \cdots, \sigma_{m}$ are the reachability indices of the precompensator remainder $\bar{v}$, then $\sum_{i=1}^{m} \sigma_{i} \geqq \sum_{i=1}^{m} v_{i}$.

Proof. Let $d_{1}, \cdots, d_{m}$ be a proper strictly polynomial basis for ker. $\pi^{-} \bar{f}$ and write ker $\pi^{-} \bar{f}=D \Omega^{-} U$ where $D=\left[d_{1}, \cdots, d_{m}\right]$. Then the matrix $D_{1}:=z^{-1} D$ is also polynomial and $D_{1}^{-1}$ is causal (see Proposition 6.18 ). Below we shall use the same notation interchangeably for matrices and their associated $\Lambda K$-linear maps. Let $L: \Lambda U \rightarrow \Lambda U$ be any static $\Lambda K$-linear map such that $L+D_{1}^{-1}$ is bicausal. Consider the bicausal pre- compensator $\bar{l}:=\left(L+D_{1}^{-1}\right)^{-1}$. If $\bar{v}$ is any precompensator remainder for $\bar{l}$, then $\bar{v}^{-1}=\bar{l}^{-1}-\bar{\rho} \bar{f}=L+D_{1}^{-1}-\bar{\rho} \bar{f}$ for some causal map $\bar{\rho}$. By Lemma 
$7.4, \bar{v}$ has the same reachability indices as $\bar{v}^{-1}$ and the latter has the same reachability indices as $D_{1}^{-1}-\bar{\rho} \bar{f}$ Now, we have

$$
D_{1}^{-1}-\bar{\rho} \cdot \bar{f}=\left(I-\bar{\rho} \cdot \bar{f} \cdot D_{1}\right) D_{1}^{-1}=\bar{I}^{*} \cdot D_{1}^{-1}
$$

where $\bar{l}^{*}=I-\bar{\rho} \cdot \bar{f} \cdot D_{1}$ is bicausal because the composite $\bar{f} \cdot D_{1}$ is strictly causal, the latter following since ker $\pi^{-} \bar{f} \cdot D_{1}=D_{1}^{-1} \operatorname{ker} \pi^{-} \bar{f}=D_{1}^{-1}\left(z D_{1}\right) \Omega^{-} U=z \Omega^{-} U$. Let $\bar{l}^{*} D_{1}^{-1}=P \cdot Q^{-1}$ be a coprime fraction representation of $\bar{l}^{*} \cdot D_{1}^{-1}$ ( see, e.g., Heymann [1972] or Hautus and Heymann [1978] . Then clearly $P$ is nonsingular, and computing determinantal degrees gives us (because $\bar{l}^{*}$ is bicausal) that

$$
n:=\operatorname{deg} \operatorname{det} Q=\operatorname{deg} \operatorname{det} P+\operatorname{deg} \operatorname{det} D_{1} \geqq \operatorname{deg} \operatorname{det} D_{1}
$$

since $n$ equals the sum of the reachability indices of the i/o map $P \cdot Q^{-1}$ the proof is complete. $\square$

Note. (added in proof). The reader is also referred to Emre and Hautus [1980], where certain solvability conditions for rational matrix equations are given that are related to the causal factorization problem.

\section{REFERENCES}

F. M. BRASH and J. B. PEARSON [1970], Pole placement using dynamic compensators, IEEE Trans. Automat. Control, AC-15, pp. 34-43.

A. E. ECKBERG, JR. [1974], A characterization of linear systems via polynomial matrices and module theory MIT Electronic Systems Laboratory Rep. ESL-R-528, Mass. Inst. of Tech., Cambridge, MA.

E. EMRE and M. L. J. HAUTUS [1980], A polynomial characterization of $((t, \mathscr{P}))$-invariant and reachability subspaces, this Journal, 18, pp. $420-436$.

P. L. FALB and W. A. WOLOVICH [1967], Decoupling in the design and synthesis of multivariable control systems, IEEE Trans. Automat. Control, AC- 12, pp. $651-659$.

G. D. FORNEY, JR. [1975], Minimal bases of rational vector spaces, with applications to multivariable linear systems, SIAM J. Control, 13, pp. 493-520.

P. A. FUHRMANN [1976], Algebraic system theory: an analyst's point of view, J. Franklin Inst., 301, pp 521 - 540

- [1979], Linear feedback via polynomial models, Int. J. Control, to appear.

E. G. GILBERT [1969], The decoupling of multivariable systems by state feedback, SIAM J. Control, 7, pp. 50-64.

W. H. GREUB [1967], Linear Algebra, 3rd edition, Springer Verlag, Berlin.

M. L. J. HAUTUS and M. HEYMANN [1978], Linear feedback-an algebraic approach, this Journal, 16, pp. 83-105.

M. HEYMANN [1968], Comments on pole assignment in multi-input controllable linear systems, IEEE Trans. Automat. Control, AC-13, pp. 748-749.

M. HEYMANN [1972], Structure and realization problems in the theory of dynamical systems, Lecture Notes, International Center for Mechanical Sciences, Udine, Italy; also Springer-Verlag, New York, 1975.

R. E. KALMAN, P.L. FALB AND M. A. ARBIB [1969], Topics in mathematical system theory, McGraw Hill, New York.

D. G. LUENBERGER [1966], Observers for multivariable systems, IEEE Trans. Automat. Control, Ac-11, pp. $190-197$.

C. C. MACDUFFEE [1934], The Theory of Matrices, Chelsea, New York.

A. S. MORSE [1975], System invariants under feedback and cascade control, Proceedings of the conference on mathematical systems theory, Udine, Italy, pp. 61 - 74; Lecture Notes in Economics and Mathematical Systems 131, Springer Verlag, Berlin.

A. S. MORSE and W. M. WONHAM [1970], Decoupling and pole assignment by dynamic compensation, SIAM J. Control, 8, pp. $317-337$.

H. F. MÜNZER and D. PRÄTZEL-WOLTERS [1979a], Minimal bases of polynomial modules, structural indices and Brunovsky-transformations, Int. J. Control, 30, pp. 291-318.

- [1979b], Geometric and moduletheoretic approach to linear systems, Part 1: basic categories and functors, Proceedings of the Delft Conference on Systems and Networks, July.

- [1979c] Geometric and moduletheoretic approach to linear systems, Part 2: moduletheoretic characterization of reachability subspaces, Internal report, Universität Bremen, Bremen, Germany.

H. H. ROSENGROCK [1970], State space and multivariable theory, Nelson, London.

J. D. SIMON and S. K. MITTER [1968], $A$ theory of modal control, Information and Control, 13, pp. $316-353$.

W. A. WOLOVICH [1974], Linear multivariable systems, Applied Mathematical Sciences Series, 11, SpringerVerlag, New York. 
W. M. WONHAM [1967], On pole assignment in multi-input controllable linear systems, IEEE Trans. Automat. Control. AC-12, pp. 660-665.

- [1979] Linear Multivariable Control: A Geometric Approach, 2nd ed., Springer-Verlag, New York.

W. M. WONHAM and A. S. Morse [1970], Decoupling and pole assignment in linear multivariable systems: A geometric approach, SIAM J. Control, 8, pp. 1 - 18.

B. F. WYMAN [1972], Linear systems over commutative rings, Lecture notes, Stanford Univ., Stanford, CA. 